\title{
THE BIVARIATE EXTENSION OF AMOROSO DISTRIBUTION
}

\author{
David Sam Jayakumar ${ }^{1 \ddagger}$, A Sulthan², and W Samuel ${ }^{3}$ \\ 1Jamal Institute of Management, Tiruchirappalli - 620020 South India, India, samjaya77@gmail.com \\ 2Jamal Institute of Management, Tiruchirappalli - 620020 South India, India, sulthan90@gmail.com \\ 3Jamal Institute of Management, Tiruchirappalli - 620020 South India, India, wsamuel365@gmail.com \\ ‡corresponding author
}

\section{Indonesian Journal of Statistics and Its Applications (elSSN:2599-0802) Vol 4 No 2 (2020), 261 - 283}

Copyright (C) 2020 David Sam Jayakumar, A Sulthan, and W Samuel. This is an open-access article distributed under the Creative Commons Attribution License, which permits unrestricted use, distribution, and reproduction in any medium, provided the original work is properly cited.

\begin{abstract}
This paper introduces the bivariate extension of the amoroso distribution and its density function is expressed in terms of hyper-geometric function. The standard amoroso distribution, cumulative distribution functions, conditional distributions, and its moments are also derived. The Product moments, Co-variance, correlations, and Shannon's differential entropy are also shown. Moreover, the generating functions such as moment, Cumulant, Characteristic functions are expressed in Fox-wright function, and the Survival, hazard, and Cumulative hazard functions are also computed. The special cases of the bivariate amoroso distribution are also discussed and nearly 780 bivariate mixtures of distributions can be derived. Finally, the two-dimensional probability surfaces are visualized for the selected special cases and we also showed the estimation of parameters by the method of maximum likelihood approach, and the constrained maximum likelihood approach is also computed by using Non-linear Programming with a numerical application.
\end{abstract}

Keywords: amoroso distribution, constrained maximum likelihood approach, Fox-wright function, hypergeometric function, probability surface parameters.

* Received Oct 2019; Accepted Jun 2020; Published online on Jul 2020 


\section{Some Preliminaries}

Explicit expressions for the PDF of bivariate amoroso distribution and the Calculation of constants, generating functions and parameter estimation of the distribution involve several special functions (Prudnikov et al., 1986) \& (Gradshteyn \& Ryzhik, 2014) and they are given as follows:

1. The Generalized hyper geometric function of order $p$ and $q$ is defined as

$$
{ }_{p} F_{q}\left(a_{1}, a_{2}, \mathrm{~K}, a_{p} ; b_{1}, b_{2}, \mathrm{~K}, b_{q} ; x\right)=\sum_{k=0}^{\infty} \frac{\left(a_{1}\right)_{k}\left(a_{2}\right)_{k} \mathrm{~K}\left(a_{p}\right)_{k}}{\left(b_{1}\right)_{k}\left(b_{2}\right)_{k} \mathrm{~K}\left(b_{q}\right)_{k}}\left(\frac{x^{k}}{k !}\right)
$$

2. The Gauss hyper geometric function is defined as

$$
{ }_{2} F_{1}\left(a_{1}, a_{2} ; b_{1} ; x\right)=\sum_{k=0}^{\infty} \frac{\left(a_{1}\right)_{k}\left(a_{2}\right)_{k}}{\left(b_{1}\right)_{k}}\left(\frac{x^{k}}{k !}\right)
$$

3. The Confluent hyper geometric function is defined as

$$
{ }_{1} F_{1}\left(a_{1} ; b_{1} ; x\right)=\sum_{k=0}^{\infty} \frac{\left(a_{1}\right)_{k}}{\left(b_{1}\right)_{k}}\left(\frac{x^{k}}{k !}\right)
$$

4. The rising factorial or Pochammer symbol is given as

$$
(a)_{k}=a(a+1)(a+2) \mathrm{K} \mathrm{K}(a+k-1)
$$

5. The integral representation of the Gamma function is defined by

$$
\Gamma(a)=\int_{0}^{\infty} x^{a-1} e^{-x} d x
$$

6. The integral representation of the lower incomplete Gamma function is defined by

$$
\gamma(a, b x)=\int_{0}^{x} t^{a-1} e^{-b t} d t
$$

7. The Binomial expression of the following series is given by

$$
(1+x)^{n}=\sum_{k=0}^{n}\left(\begin{array}{l}
n \\
k
\end{array}\right) x^{k}
$$

8. The Fox-wright function of order $p$ and $q$ is defined as

$$
{ }_{p} \Psi_{q}\left[\begin{array}{cccc}
\left(a_{1}, A_{1}\right) & \left(a_{2}, A_{2}\right) & \mathrm{L} & \left(a_{p}, A_{p}\right) \\
\left(b_{1}, B_{1}\right) & \left(b_{2}, B_{2}\right) & \mathrm{L} & \left(b_{q}, B_{q}\right)
\end{array}\right] x=\sum_{k=0}^{\infty} \frac{\Gamma\left(a_{1}+A_{1} k\right) \Gamma\left(a_{2}+A_{2} k\right) \mathrm{K} \Gamma\left(a_{p}+A_{p} k\right)}{\Gamma\left(b_{1}+B_{1} k\right) \Gamma\left(b_{2}+B_{2} k\right) \mathrm{K} \Gamma\left(b_{q}+B_{q} k\right)}\left(\frac{x^{k}}{k !}\right)
$$

9. The first derivative of the Gamma function is di-gamma function which is given as

$$
\psi(x)=\frac{d}{d x}(\log \Gamma(x))
$$

10. The first derivative of the hyper-geometric function ${ }_{0} F_{1}$ with respect ' $a_{1}$ ' is given as

$$
{ }_{0} F_{1(a)}^{\prime}\left(\alpha ; \frac{\lambda^{2}}{\left(1-\lambda^{2}\right)^{2}}\left(\frac{x_{i}-a_{1}}{b_{1}}\right)^{\beta_{1}}\left(\frac{y_{i}-a_{2}}{b_{2}}\right)^{\beta_{2}}\right)=\sum_{k=0}^{\infty} \frac{1}{(\alpha)_{k} k !}\left(\frac{\lambda^{2 k}}{\left(1-\lambda^{2}\right)^{2 k}}\left(-\left(\frac{\beta_{1} k}{x_{i}-a_{1}}\right)\left(\frac{x_{i}-a_{1}}{b_{1}}\right)^{\beta_{1} k}\right)\left(\frac{y_{i}-a_{2}}{b_{2}}\right)^{\beta_{2} k}\right)
$$


11. The first derivative of the hyper-geometric function ${ }_{0} F_{1}$ with respect ' $a_{2}$ ' is given as ${ }_{0} F_{1\left(a_{2}\right)}^{\prime}\left(\alpha ; \frac{\lambda^{2}}{\left(1-\lambda^{2}\right)^{2}}\left(\frac{x_{i}-a_{1}}{b_{1}}\right)^{\beta_{1}}\left(\frac{y_{i}-a_{2}}{b_{2}}\right)^{\beta_{2}}\right)=\sum_{k=0}^{\infty} \frac{1}{(\alpha)_{k} k !}\left(\frac{\lambda^{2 k}}{\left(1-\lambda^{2}\right)^{2 k}}\left(\frac{x_{i}-a_{1}}{b_{1}}\right)^{\beta_{1} k}\left(-\left(\frac{\beta_{2} k}{y_{i}-a_{2}}\right)\left(\frac{y_{i}-a_{2}}{b_{2}}\right)^{\beta_{2} k}\right)\right)$

12. The first derivative of the hyper-geometric function ${ }_{0} F_{1}$ with respect ' $b_{1}$ ' is given as

${ }_{0} F_{1\left(b_{1}\right)}^{\prime}\left(\alpha ; \frac{\lambda^{2}}{\left(1-\lambda^{2}\right)^{2}}\left(\frac{x_{i}-a_{1}}{b_{1}}\right)^{\beta_{1}}\left(\frac{y_{i}-a_{2}}{b_{2}}\right)^{\beta_{2}}\right)=\sum_{k=0}^{\infty} \frac{1}{(\alpha)_{k} k !}\left(\frac{\lambda^{2 k}}{\left(1-\lambda^{2}\right)^{2 k}}\left(-\left(\frac{\beta_{1} k}{b_{1}}\right)\left(\frac{x_{i}-a_{1}}{b_{1}}\right)^{\beta_{1} k}\right)\left(\frac{y_{i}-a_{2}}{b_{2}}\right)^{\beta_{2} k}\right)$

13. The first derivative of the hyper-geometric function ${ }_{0} F_{1}$ with respect ' $b_{2}$ ' is given as

${ }_{0} F_{1(2)}^{\prime}\left(\alpha ; \frac{\lambda^{2}}{\left(1-\lambda^{2}\right)^{2}}\left(\frac{x_{i}-a_{1}}{b_{1}}\right)^{\beta_{1}}\left(\frac{y_{i}-a_{2}}{b_{2}}\right)^{\beta_{2}}\right)=\sum_{k=0}^{\infty} \frac{1}{(\alpha)_{k} k !}\left(\frac{\lambda^{2 k}}{\left(1-\lambda^{2}\right)^{2 k}}\left(\frac{x_{i}-a_{1}}{b_{1}}\right)^{\beta_{1} k}\left(-\left(\frac{\beta_{2} k}{b_{2}}\right)\left(\frac{y_{i}-a_{2}}{b_{2}}\right)^{\beta_{2} k}\right)\right)$

14. The first derivative of the hyper-geometric function ${ }_{0} F_{1}$ with respect ' $\alpha$ ' is given as

${ }_{0} F_{1(\alpha)}^{\prime}\left(\alpha ; \frac{\lambda^{2}}{\left(1-\lambda^{2}\right)^{2}}\left(\frac{x_{i}-a_{1}}{b_{1}}\right)^{\beta_{1}}\left(\frac{y_{i}-a_{2}}{b_{2}}\right)^{\beta_{2}}\right)=\sum_{k=0}^{\infty} \frac{(\Psi(\alpha)-\Psi(\alpha+k))}{(\alpha)_{k} k !}\left(\frac{\lambda^{2 k}}{\left(1-\lambda^{2}\right)^{2 k}}\left(\frac{x_{i}-a_{1}}{b_{1}}\right)^{\beta_{1} k}\left(\frac{y_{i}-a_{2}}{b_{2}}\right)^{\beta_{2} k}\right)$

15. The first derivative of the hyper-geometric function ${ }_{0} F_{1}$ with respect ' $\beta_{1}$ ' is given as

${ }_{0} F_{1\left(\beta_{1}\right)}^{\prime}\left(\alpha ; \frac{\lambda^{2}}{\left(1-\lambda^{2}\right)^{2}}\left(\frac{x_{i}-a_{1}}{b_{1}}\right)^{\beta_{1}}\left(\frac{y_{i}-a_{2}}{b_{2}}\right)^{\beta_{2}}\right)=\sum_{k=0}^{\infty} \frac{1}{(\alpha)_{k} k !}\left(\frac{\lambda^{2 k}}{\left(1-\lambda^{2}\right)^{2 k}}\left(k\left(\frac{x_{i}-a_{1}}{b_{1}}\right)^{\beta_{1} k} \log \left(\frac{x_{i}-a_{1}}{b_{1}}\right)\right)\left(\frac{y_{i}-a_{2}}{b_{2}}\right)^{\beta_{2} k}\right)$

16. The first derivative of the hyper-geometric function ${ }_{0} F_{1}$ with respect ' $\beta_{2}$ ' is given as

${ }_{0} F_{\mathrm{L}\left(\beta_{2}\right)}^{\prime}\left(\alpha ; \frac{\lambda^{2}}{\left(1-\lambda^{2}\right)^{2}}\left(\frac{x_{i}-a_{1}}{b_{1}}\right)^{\beta_{1}}\left(\frac{y_{i}-a_{2}}{b_{2}}\right)^{\beta_{2}}\right)=\sum_{k=0}^{\infty} \frac{1}{(\alpha)_{k} k !}\left(\frac{\lambda^{2 k}}{\left(1-\lambda^{2}\right)^{2 k}}\left(\frac{x_{i}-a_{1}}{b_{1}}\right)^{\beta_{1} k}\left(k\left(\frac{y_{i}-a_{2}}{b_{2}}\right)^{\beta_{2} k} \log \left(\frac{y_{i}-a_{1}}{b_{1}}\right)\right)\right)$

17. The first derivative of the hyper-geometric function ${ }_{0} F_{1}$ with respect ' $\lambda$ ' is given as

${ }_{0} F_{1(\lambda)}^{\prime}\left(\alpha ; \frac{\lambda^{2}}{\left(1-\lambda^{2}\right)^{2}}\left(\frac{x_{i}-a_{1}}{b_{1}}\right)^{\beta_{1}}\left(\frac{y_{i}-a_{2}}{b_{2}}\right)^{\beta_{2}}\right)=\sum_{k=0}^{\infty} \frac{1}{(\alpha)_{k} k !}\left(-\frac{2 k \lambda^{2 k}\left(1+\lambda^{2}\right)}{\left(\lambda^{3}-\lambda\right)\left(1-\lambda^{2}\right)^{2 k}}\left(\frac{x_{i}-a_{1}}{b_{1}}\right)^{\beta_{1} k}\left(\frac{y_{i}-a_{2}}{b_{2}}\right)^{\beta_{2} k}\right)$

\section{Introduction to Amoroso Distribution}

The Amoroso (generalized gamma, Stacy-Mihram) distribution is a generalized distribution with four parameters, continuous, univariate, unimodal probability density and semi-infinite range. The Amoroso distribution was originally developed to model lifetimes. It occurs as the Weibullization of the standard gamma distribution and, with integer $\alpha$, in extreme value statistics. The Amoroso distribution is itself a limiting form of various more general distributions, most notable the generalized beta and generalized beta prime distributions. A useful and important property of the Amoroso distribution is that many common and interesting probability distributions occur as special cases or limits. Informally, an interesting distribution" is one that has acquired a name, which generally indicates that the distribution is the solution to one or more interesting problems. This 
provides a convenient method for systemizing a significant fraction of the probability distributions that are encountered in practice, provides a consistent parameterization for those distributions, and obviates the need to enumerate the properties (mean, mode, variance, entropy and so on) of each and every specialization. The Amoroso, gamma family of distributions are considered to be the generalized family of semi-infinite support distributions. The applications of the generalized distributions are extensively studied and the authors shown some of the important and interesting applications are as follows.

\subsection{Application of some Generalized Distributions}

Moghaddam et al. (2019) argued that a stochastic model of economic exchange, whose steady-state distribution is a Generalized Beta Prime (also known as GB2), and some unique properties of the latter, are the reason for GB2's success in describing wealth/income distributions. Bhatti et al. (2019) presented a generalized log Burr III (GLBIII) distribution developed on the basis of a generalized log Pearson differential equation (GLPE). Oladipo (2019) investigated the polynomials whose coefficients are generalized distribution. Clementi et al. (2008) proposed the k-generalized distribution as a model for describing the distribution and dispersion of income within a population. Ramos \& Louzada (2018) presented a Bayesian reference analysis for the generalized gamma distribution by using a reference prior, which has important properties such as one-to-one invariance under reparametrization, consistent marginalization, consistent sampling and leads to a proper posterior density. Tripathi et al., (2018) introduced a generalized inverse x-gamma distribution (GIXGD) as the generalized version of the inverse $x$-gamma distribution. The proposed model exhibits the pattern of non-monotone hazard rate and belongs to family of positively skewed models. Mansoor et al. (2019) introduced a three-parameter extension of the exponential distribution which contains sub-models as the exponential, logistic-exponential and Marshall-Olkin exponential distributions. The Generalized gamma (GG) distribution plays an important role in statistical analysis. For this distribution, Ramos et al. (2017) derived non-informative priors using formal rules, such as Jeffreys prior, maximal data information prior and reference priors. Progri (2016) discussed the exponential generalized Beta distribution (EGBD). For the EGBD model the author provided the closed form expression of the cumulative distribution function (cdf), statistics for special cases and the computation of the mean and variance for the general case. VedoVatto et al. (2016) introduced a new four-parameter model called the Exponentiated Generalized Nadarajah-Haghighi $(E G N H)$ distribution in order to verify this requirement. They proved that its hazard rate function can be constant, decreasing, increasing, upside-down bathtub and bathtubshape. Merovci (2014) discussed the generalized Rayleigh distribution using the quadratic rank transmutation map studied by Shaw et al. to develop a transmuted generalized Rayleigh distribution. Potdar \& Shirke (2013) introduced a generalized inverted scale family of distributions. Cordeiro et al. (2012) proposed and studied the Kumaraswamy generalized half-normal distribution for modeling skewed positive data. The half-normal and generalized half-normal distributions are special cases of the new 
model. Hamedani (2011) presented the characterizations of a continuous univariate distribution due to Shakil, Kibria and Singh (SKS), based on a simple relationship between two truncated moments. And also pointed out that some special cases of the SKS distribution can be characterized based on the hazard function. Nassar \& Nada (2011) proposed a new distribution called the beta-generalized Pareto. Several properties of this distribution are presented. Abd-Elfattah et al. (2010) obtained the tables of critical values of modified Kolmogorov-Smirnov (KS) test, Cramer-Von Mises (CVM) test, AndersonDarling (AD) and Watson test for generalized Frechet distribution with unknown parameters. The sampling distributions for these tests statistics are investigated. Here, they used Monte Carlo and Pearson system techniques to create tables of critical values for such situations. Scott et al. (2011) demonstrated a recursive method for obtaining the moments of the generalized hyperbolic distribution. The method is readily programmable for numerical evaluation of moments. For low order moments we also give an alternative derivation of the moments of the generalized hyperbolic distribution. Here, these versatile applications of the generalized distributions motivated the authors to extend the univariate Amoroso distribution to its bivariate case and the characteristics and properties of the proposed distribution are extensively studied in the following sections.

\subsection{Bivariate Amoroso distribution}

Definition 1.1: Let $X$ and $Y$ be the random variables followed Bivariate Amoroso distribution with location $\left(a_{1}, a_{2}\right)$, scale $\left(b_{1}, b_{2}\right)$ and shape $\left(\alpha, \beta_{1}, \beta_{2}\right)$ parameters with a dependency co-efficient $(\lambda)$, then the density function of the distribution (Amoroso \& Mignotte, 1996) is defined as

$$
f_{X, Y}(x, y)=\frac{\left|\beta_{1}\right|\left|\beta_{2}\right|}{b_{1} b_{2} \Gamma^{2}(\alpha)\left(1-\lambda^{2}\right)^{\alpha}}\left(\begin{array}{l}
\left(\frac{x-a_{1}}{b_{1}}\right)^{\alpha \beta_{1}-1}\left(\frac{y-a_{2}}{b_{2}}\right)^{\alpha \beta_{2}-1} e^{-\frac{1}{1-\lambda^{2}}\left(\left(\frac{x-a_{1}}{b_{1}}\right)^{\beta_{1}}+\left(\frac{y-a_{2}}{b_{2}}\right)^{\beta_{2}}\right)} \\
\times_{0} F_{1}\left(\alpha ; \frac{\lambda^{2}}{\left(1-\lambda^{2}\right)^{2}}\left(\frac{x-a_{1}}{b_{1}}\right)^{\beta_{1}}\left(\frac{y-a_{2}}{b_{2}}\right)^{\beta_{2}}\right)
\end{array}\right)-
$$

where $a_{1} \leq x<\infty, a_{2} \leq y<\infty, a_{1}, a_{2}, b_{1}, b_{2}, \alpha, \beta_{1}, \beta_{2}>0,-1<\lambda<+1$ and ${ }_{0} F_{1}()$ is the hyper geometric function respectively.

Theorem 1.2: If $Z_{x}=\left(X-a_{1}\right) / b_{1}$ and $Z_{y}=\left(Y-a_{2}\right) / b_{2}$ then the Bivariate Amoroso distribution was transformed into standard Bivariate Amoroso distribution with shape $\left(\alpha, \beta_{1}, \beta_{2}\right)$ parameters with a dependency co-efficient $(\lambda)$, then it's density function is given as 


$$
f_{Z_{X}, Z_{Y}}\left(z_{x}, z_{y}\right)=\frac{\left|\beta_{1}\right|\left|\beta_{2}\right|}{\Gamma^{2}(\alpha)\left(1-\lambda^{2}\right)^{\alpha}}\left(\begin{array}{l}
\left.\left(z_{x}\right)^{\alpha \beta_{1}-1}\left(z_{y}\right)^{\alpha \beta_{2}-1} e^{-\frac{1}{1-\lambda^{2}}\left(\left(z_{x}\right)^{\beta_{1}}+\left(z_{y}\right)^{\beta_{2}}\right.}\right) \\
\times{ }_{0} F_{1}\left(\alpha ; \frac{\lambda^{2}}{\left(1-\lambda^{2}\right)^{2}}\left(z_{x}\right)^{\beta_{1}}\left(z_{y}\right)^{\beta_{2}}\right)
\end{array}\right)-
$$

Theorem 1.3: The cumulative distribution function of the Bivariate Amoroso distribution is defined by

$$
F_{X, Y}(x, y)=\frac{1}{\Gamma^{2}(\alpha)\left(1-\lambda^{2}\right)^{\alpha}} \sum_{k=0}^{\infty} \frac{1}{(\alpha)_{k} k !}\left(\frac{\lambda^{2 k}}{\left(1-\lambda^{2}\right)^{2 k}} \gamma\left(\alpha+k, \frac{\left(\left(x-a_{1}\right) / b_{1}\right)^{\beta_{1}}}{1-\lambda^{2}}\right) \gamma\left(\alpha+k, \frac{\left(\left(y-a_{2}\right) / b_{2}\right)^{\beta_{2}}}{1-\lambda^{2}}\right)\right)-
$$

Where $\gamma()$ is the lower incomplete Gamma function.

Proof: Let the cumulative distribution function of a bivariate distribution is

$$
\left.\begin{array}{c}
F_{X, Y}(x, y)=\int_{a_{1}}^{x} \int_{a_{2}}^{y} f(U, V) d U d V \\
\left.F_{X, Y}(x, y)=\frac{\left|\beta_{1}\right|\left|\beta_{2}\right|}{b_{1} b_{2} \Gamma^{2}(\alpha)\left(1-\lambda^{2}\right)^{\alpha}}\left(\int_{a_{1} a_{2}}^{x} \int_{x_{0}}^{x} F_{1}\left(\frac{U-a_{1}}{b_{1}}\right)^{\alpha \beta_{1}-1}\left(\frac{V-a_{2}}{b_{2}}\right)^{\alpha \beta_{2}-1} e^{\left.-\frac{1}{1-\lambda^{2}}\left(\frac{\lambda^{2}}{b_{1}}\right)^{2}+\left(\frac{U-a_{1}}{b_{1}}\right)^{\beta_{1}}\left(\frac{V-\lambda_{2}}{b_{2}}\right)^{\beta_{2}}\right)}\right) d U d V-\left(1 . a_{2}\right)^{\beta_{2}}\right)
\end{array}\right]
$$

By Setting $z_{x}=\left(\left(U-a_{1}\right) / b_{1}\right)^{\beta_{1}}, z_{y}=\left(\left(V-a_{2}\right) / b_{2}\right)^{\beta_{1}}$ and expand the generalized hypergeometric function ${ }_{0} F_{1}()$, then the integral expression of (1.4) becomes

$$
=\frac{1}{\Gamma^{2}(\alpha)\left(1-\lambda^{2}\right)^{\alpha}} \sum_{k=0}^{\infty} \frac{1}{(\alpha)_{k} k !}\left(\frac{\lambda^{2 k}}{\left(1-\lambda^{2}\right)^{2 k}} \int_{0}^{\left(\left(x-a_{1}\right) / b_{1}\right)^{\beta_{1}}}\left(z_{x}\right)^{\alpha+k-1} e^{-\frac{z_{x}}{1-\lambda^{2}}} d z_{x} \int_{0}^{\left(\left(y-a_{2}\right) / b_{2}\right)^{\beta_{2}}}\left(z_{y}\right)^{\alpha+k-1} e^{-\frac{z_{y}}{1-\lambda^{2}}} d z_{y}\right)
$$

Now integrate (1.4), then the final form of CDF as

$$
F_{X, Y}(x, y)=\frac{1}{\Gamma^{2}(\alpha)\left(1-\lambda^{2}\right)^{\alpha}} \sum_{k=0}^{\infty} \frac{1}{(\alpha)_{k} k !}\left(\frac{\lambda^{2 k}}{\left(1-\lambda^{2}\right)^{2 k}} \gamma\left(\alpha+k, \frac{\left(\left(x-a_{1}\right) / b_{1}\right)^{\beta_{1}}}{1-\lambda^{2}}\right) \gamma\left(\alpha+k, \frac{\left(\left(y-a_{2}\right) / b_{2}\right)^{\beta_{2}}}{1-\lambda^{2}}\right)\right)
$$

where $\gamma()$ is the lower incomplete Gamma function.

\section{Marginal and Conditional distributions}

Theorem 2.1: The marginal distribution of $X$ for (1.1) is the univariate amoroso distribution and it's density function is given as 


$$
f_{X}(x)=\frac{\left|\beta_{1}\right|}{b_{1} \Gamma(\alpha)}\left(\frac{x-a_{1}}{b_{1}}\right)^{\alpha \beta_{1}-1} e^{-\left(\frac{x-a_{1}}{b_{1}}\right)^{\beta_{1}}}
$$

where $a_{1} \leq x<\infty, a_{1}, b_{1}, \alpha, \beta_{1}>0$

Proof: The marginal distribution of Bivariate Amoroso distribution of $X$ is derived as

$$
\begin{gathered}
f_{X}(x)=\int_{a_{2}}^{\infty} f_{X, Y}(x, y) d y \\
=\frac{\left|\beta_{1}\right|\left|\beta_{2}\right|}{b_{1} b_{2} \Gamma^{2}(\alpha)\left(1-\lambda^{2}\right)^{\alpha}} \int_{a_{2}}^{\infty}\left(\begin{array}{l}
\left.\left(\frac{x-a_{1}}{b_{1}}\right)^{\alpha \beta_{1}-1}\left(\frac{y-a_{2}}{b_{2}}\right)^{\alpha \beta_{2}-1} e^{-\frac{1}{1-\lambda^{2}}\left(\left(\frac{x-a_{1}}{b_{1}}\right)^{\beta_{1}}+\left(\frac{y-a_{2}}{b_{2}}\right)^{\beta_{2}}\right)}\right) \\
\times{ }_{0} F_{1}\left(\alpha ; \frac{\lambda^{2}}{\left(1-\lambda^{2}\right)^{2}}\left(\frac{x-a_{1}}{b_{1}}\right)^{\beta_{1}}\left(\frac{y-a_{2}}{b_{2}}\right)^{\beta_{2}}\right)
\end{array}\right) d y
\end{gathered}
$$

From (2.2) set $S=\left(y-a_{2}\right) / b_{2}$,expand the hyper-geometric function and integrate it, then the final result is found to be

$$
\begin{gathered}
f_{X}(x)=\frac{\left|\beta_{1}\right|}{b_{1} \Gamma(\alpha)}\left(\frac{x-a_{1}}{b_{1}}\right)^{\alpha \beta_{1}-1} e^{-\left(\frac{x-a_{1}}{b_{1}}\right)^{\beta_{1}}} \\
\text { where } a_{1} \leq x<\infty, a_{1}, b_{1}, \alpha, \beta_{1}>0
\end{gathered}
$$

Theorem 2.2: The marginal distribution of $Y$ for (1.1) is the univariate amoroso distribution and it's density function is given as

$$
\begin{gathered}
f_{Y}(y)=\frac{\left|\beta_{2}\right|}{b_{2} \Gamma(\alpha)}\left(\frac{y-a_{2}}{b_{2}}\right)^{\alpha \beta_{2}-1} e^{-\left(\frac{y-a_{2}}{b_{2}}\right)^{\beta_{2}}} \\
\text { where } a_{2} \leq x<\infty, a_{2}, b_{2}, \alpha, \beta_{2}>0
\end{gathered}
$$

Proof: The marginal distribution of Bivariate Amoroso distribution of $Y$ is computed as

$$
\begin{gathered}
f_{Y}(y)=\int_{a_{1}}^{\infty} f_{X, Y}(x, y) d x \\
\left.=\frac{\left|\beta_{1}\right|\left|\beta_{2}\right|}{b_{1} b_{2} \Gamma^{2}(\alpha)\left(1-\lambda^{2}\right)^{\alpha}} \int_{a_{2}}^{\infty}\left(\frac{x-a_{1}}{b_{1}}\right)^{\alpha \beta_{1}-1}\left(\frac{y-a_{2}}{b_{2}}\right)^{\alpha \beta_{2}-1} e^{-\frac{1}{1-\lambda^{2}}\left(\left(\frac{x-a_{1}}{b_{1}}\right)^{\beta_{1}}+\left(\frac{y-a_{2}}{b_{2}}\right)^{\beta_{2}}\right)}\right) d x \\
\times_{0} F_{1}\left(\alpha ; \frac{\lambda^{2}}{\left(1-\lambda^{2}\right)^{2}}\left(\frac{x-a_{1}}{b_{1}}\right)^{\beta_{1}}\left(\frac{y-a_{2}}{b_{2}}\right)^{\beta_{2}}\right)
\end{gathered}
$$

From (2.4) similarly set $S=\left(x-a_{2}\right) / b_{2}$, expand the hyper-geometric function and integrate it, then the final result is found to be

$$
\begin{array}{r}
f_{Y}(y)=\frac{1}{\Gamma(\alpha)}\left|\frac{\beta_{2}}{b_{2}}\right|\left(\frac{y-a_{2}}{b_{2}}\right)^{\alpha \beta_{2}-1} e^{-\left(\frac{y-a_{2}}{b_{2}}\right)^{\beta_{2}}} \\
\text { where } a_{2} \leq x<\infty, a_{2}, b_{2}, \alpha, \beta_{2}>0
\end{array}
$$


Theorem 2.3: The Probability density function of Conditional Amoroso distribution of $X$ on $Y$ is

$$
\begin{gathered}
f_{X / Y}(x / y)=\frac{\left|\beta_{1}\right|}{b_{1} \Gamma(\alpha)\left(1-\lambda^{2}\right)^{\alpha}}\left(\begin{array}{l}
\left(\frac{x-a_{1}}{b_{1}}\right)^{\alpha \beta_{1}-1} e^{-\frac{1}{1-\lambda^{2}}\left(\left(\frac{x-a_{1}}{b_{1}}\right)^{\beta_{1}}+\lambda^{2}\left(\frac{y-a_{2}}{b_{2}}\right)^{\beta_{2}}\right)} \\
\times_{0} F_{1}\left(\alpha ; \frac{\lambda^{2}}{\left(1-\lambda^{2}\right)^{2}}\left(\frac{x-a_{1}}{b_{1}}\right)^{\beta_{1}}\left(\frac{y-a_{2}}{b_{2}}\right)^{\beta_{2}}\right)
\end{array}\right) \\
\text { where } a_{1} \leq x<\infty, a_{1}, b_{1}, \alpha, \beta_{1}>0
\end{gathered}
$$

Proof: It is obtained from the fact $f_{X / Y}(x / y)=\frac{f_{X, Y}(x, y)}{f_{Y}(y)}$

Theorem 2.4: The Probability density function of Conditional Amoroso distribution of $Y$ on $X$ is

$$
\begin{gathered}
f_{Y / X}(y / x)=\frac{\left|\beta_{2}\right|}{b_{2} \Gamma(\alpha)\left(1-\lambda^{2}\right)^{\alpha}}\left(\begin{array}{l}
\left(\frac{y-a_{2}}{b_{2}}\right)^{\alpha \beta_{2}-1} e^{\left.-\frac{1}{1-\lambda^{2}}\left(\frac{y-a_{2}}{b_{2}}\right)^{\beta_{2}}+\lambda^{2}\left(\frac{x-a_{1}}{b_{1}}\right)^{\beta_{1}}\right)} \\
\times_{0} F_{1}\left(\alpha ; \frac{\lambda^{2}}{\left(1-\lambda^{2}\right)^{2}}\left(\frac{x-a_{1}}{b_{1}}\right)^{\beta_{1}}\left(\frac{y-a_{2}}{b_{2}}\right)^{\beta_{2}}\right)
\end{array}\right) \\
\text { where } a_{2} \leq x<\infty, a_{2}, b_{2}, \alpha, \beta_{2}>0
\end{gathered}
$$

Proof: It is obtained from $f_{Y / X}(y / x)=\frac{f_{X, Y}(x, y)}{f_{X}(x)}$

\section{Constants of Conditional and Bivariate Amoroso distribution}

Theorem 3.1: The $r^{\text {th }}$ order moment of the Conditional Amoroso distribution of $X$ on $Y$ is given as

$$
E_{X / Y}\left(x^{r} / y\right)=\frac{a_{1}^{r} e^{-\frac{\lambda^{2}}{1-\lambda^{2}}\left(\frac{y-a_{2}}{b_{2}}\right)^{\beta_{2}}}}{\Gamma(\alpha)} \sum_{m=0}^{r}\left(\begin{array}{l}
\left(\begin{array}{c}
r \\
m
\end{array}\right) \Gamma\left(\alpha+\left(m / \beta_{1}\right)\right) \\
\times_{1} F_{1}\left(\alpha+\left(m / \beta_{1}\right), \alpha ; \frac{\lambda^{2}}{1-\lambda^{2}}\left(\frac{y-a_{2}}{b_{2}}\right)^{\beta_{2}}\right)\left(b_{1} / a_{1}\left(1-\lambda^{2}\right)^{1 / \beta_{1}}\right)^{m}
\end{array}\right)
$$

Where ${ }_{1} F_{1}()$ is the confluent hyper geometric function.

Proof: The $r^{\text {th }}$ moment of a conditional distribution is

$$
E_{X / Y}\left(x^{r} / y\right)=\int_{a_{1}}^{\infty} x^{r} f_{X / Y}(x / y) d x
$$




$$
=\frac{\left|\beta_{1}\right|}{b_{1} \Gamma(\alpha)\left(1-\lambda^{2}\right)^{\alpha}} \int_{a_{1}}^{\infty}\left(\begin{array}{l}
\left.x^{r}\left(\frac{x-a_{1}}{b_{1}}\right)^{\alpha \beta_{1}-1} e^{-\frac{1}{1-\lambda^{2}}\left(\left(\frac{x-a_{1}}{b_{1}}\right)^{\beta_{1}}+\rho^{2}\left(\frac{y-a_{2}}{b_{2}}\right)^{\beta_{2}}\right.}\right) \\
\times_{0} F_{1}\left(\alpha ; \frac{\lambda^{2}}{\left(1-\lambda^{2}\right)^{2}}\left(\frac{x-a_{1}}{b_{1}}\right)^{\beta_{1}}\left(\frac{y-a_{2}}{b_{2}}\right)^{\beta_{2}}\right)
\end{array}\right) d x-
$$

From (3.2), Set $S=\left(\left(x-a_{1}\right) / b_{1}\right)^{\beta_{1}}$ and the write the hyper geometric function in series expansion form, and then the final result of the integration is found to be

$$
E_{X / Y}\left(x^{r} / y\right)=\frac{a_{1}^{r} e^{-\frac{\lambda^{2}}{1-\lambda^{2}}\left(\frac{y-a_{2}}{b_{2}}\right)^{\beta_{2}}}}{\Gamma(\alpha)} \sum_{m=0}^{r}\left(\begin{array}{l}
\left(\begin{array}{c}
r \\
m
\end{array}\right) \Gamma\left(\alpha+\left(m / \beta_{1}\right)\right) \\
\left.\times_{1} F_{1}\left(\alpha+\left(m / \beta_{1}\right), \alpha ; \frac{\lambda^{2}}{1-\lambda^{2}}\left(\frac{y-a_{2}}{b_{2}}\right)^{\beta_{2}}\right)\left(b_{1} / a_{1}\left(1-\lambda^{2}\right)^{1 / \beta_{1}}\right)^{m}\right)
\end{array}\right)
$$

From (3.3), If $r=1,2,3,4$ then the following Conditional moments are given as

$$
\begin{aligned}
& E_{X / Y}(x / y)=\frac{a_{1} e^{-\frac{\lambda^{2}}{1-\lambda^{2}}\left(\frac{y-a_{2}}{b_{2}}\right)^{\beta_{2}}}}{\Gamma(\alpha)} \sum_{m=0}^{1}\left(\begin{array}{l}
\left(\begin{array}{c}
1 \\
m
\end{array}\right) \Gamma\left(\alpha+\left(m / \beta_{1}\right)\right) \\
\left.\times_{1} F_{1}\left(\alpha+\left(m / \beta_{1}\right), \alpha ; \frac{\lambda^{2}}{1-\lambda^{2}}\left(\frac{y-a_{2}}{b_{2}}\right)^{\beta_{2}}\right)\left(b_{1} / a_{1}\left(1-\lambda^{2}\right)^{1 / \beta_{1}}\right)^{m}\right)
\end{array}\right) \\
& E_{X / Y}\left(x^{2} / y\right)=\frac{a_{1}^{2} e^{-\frac{\lambda^{2}}{1-\lambda^{2}}\left(\frac{y-a_{2}}{b_{2}}\right)^{\beta_{2}}}}{\Gamma(\alpha)} \sum_{m=0}^{2}\left(\begin{array}{l}
\left(\begin{array}{c}
2 \\
m
\end{array}\right) \Gamma\left(\alpha+\left(m / \beta_{1}\right)\right) \\
\left.\times_{1} F_{1}\left(\alpha+\left(m / \beta_{1}\right), \alpha ; \frac{\lambda^{2}}{1-\lambda^{2}}\left(\frac{y-a_{2}}{b_{2}}\right)^{\beta_{2}}\right)\left(b_{1} / a_{1}\left(1-\lambda^{2}\right)^{1 / \beta_{1}}\right)^{m}\right)
\end{array}\right) \\
& E_{X / Y}\left(x^{3} / y\right)=\frac{a_{1}^{3} e^{-\frac{\lambda^{2}}{1-\lambda^{2}}\left(\frac{y-a_{2}}{b_{2}}\right)^{\beta_{2}}}}{\Gamma(\alpha)} \sum_{m=0}^{3}\left(\begin{array}{l}
\left(\begin{array}{c}
3 \\
m
\end{array}\right) \Gamma\left(\alpha+\left(m / \beta_{1}\right)\right) \\
\left.\times_{1} F_{1}\left(\alpha+\left(m / \beta_{1}\right), \alpha ; \frac{\lambda^{2}}{1-\lambda^{2}}\left(\frac{y-a_{2}}{b_{2}}\right)^{\beta_{2}}\right)\left(b_{1} / a_{1}\left(1-\lambda^{2}\right)^{1 / \beta_{1}}\right)^{m}\right)
\end{array}\right. \\
& E_{X / Y}\left(x^{4} / y\right)=\frac{a_{1}^{4} e^{-\frac{\rho^{2}}{1-\rho^{2}}\left(\frac{y-a_{2}}{b_{2}}\right)^{\beta_{2}}}}{\Gamma(\alpha)} \sum_{m=0}^{4}\left(\begin{array}{l}
\left(\begin{array}{c}
4 \\
m
\end{array}\right) \Gamma\left(\alpha+\left(m / \beta_{1}\right)\right) \\
\left.\times_{1} F_{1}\left(\alpha+\left(m / \beta_{1}\right), \alpha ; \frac{\rho^{2}}{1-\rho^{2}}\left(\frac{y-a_{2}}{b_{2}}\right)^{\beta_{2}}\right)\left(b_{1} / a_{1}\left(1-\rho^{2}\right)^{1 / \beta_{1}}\right)^{m}\right)
\end{array}\right.
\end{aligned}
$$

Theorem 3.2: The $r^{\text {th }}$ conditional moment of the Conditional Amoroso distribution of $Y$ on $X$ is

$$
E_{Y / X}\left(y^{r} / x\right)=\frac{a_{2}^{r} e^{-\frac{\lambda^{2}}{1-\lambda^{2}}\left(\frac{x-a_{1}}{b_{1}}\right)^{\beta_{2}}}}{\Gamma(\alpha)} \sum_{m=0}^{r}\left(\begin{array}{l}
\left(\begin{array}{c}
r \\
m
\end{array}\right) \Gamma\left(\alpha+\left(m / \beta_{2}\right)\right) \\
\left.\times_{1} F_{1}\left(\alpha+\left(m / \beta_{2}\right), \alpha ; \frac{\lambda^{2}}{1-\lambda^{2}}\left(\frac{x-a_{1}}{b_{1}}\right)^{\beta_{1}}\right)\left(b_{2} / a_{2}\left(1-\lambda^{2}\right)^{1 / \beta_{2}}\right)^{m}\right)
\end{array}\right.
$$

Where ${ }_{1} F_{1}($ ) is the confluent hyper geometric function. 
Proof: The $r^{\text {th }}$ conditional moment of a distribution is

$$
\begin{gathered}
E_{Y / X}\left(y^{r} / x\right)=\int_{a_{2}}^{\infty} y^{r} f_{Y / X}(y / x) d y \\
=\frac{\left|\beta_{2}\right|}{b_{2} \Gamma(\alpha)\left(1-\lambda^{2}\right)^{\alpha}} \int_{a_{2}}^{\infty}\left(\begin{array}{c}
y^{r}\left(\frac{y-a_{2}}{b_{2}}\right)^{\alpha \beta_{2}-1} e^{-\frac{1}{1-\lambda^{2}}\left(\left(\frac{y-a_{2}}{b_{2}}\right)^{\beta_{2}}+\rho^{2}\left(\frac{x-a_{1}}{b_{1}}\right)^{\beta_{1}}\right)} \\
\times_{0} F_{1}\left(\alpha ; \frac{\lambda^{2}}{\left(1-\lambda^{2}\right)^{2}}\left(\frac{x-a_{1}}{b_{1}}\right)^{\beta_{1}}\left(\frac{y-a_{2}}{b_{2}}\right)^{\beta_{2}}\right)
\end{array}\right) d y
\end{gathered}
$$

From (3.9), Set $S=\left(\left(y-a_{2}\right) / b_{2}\right)^{\beta_{2}}$, write the hyper geometric functions in series expansion form and integrate it, then the final result is found to be

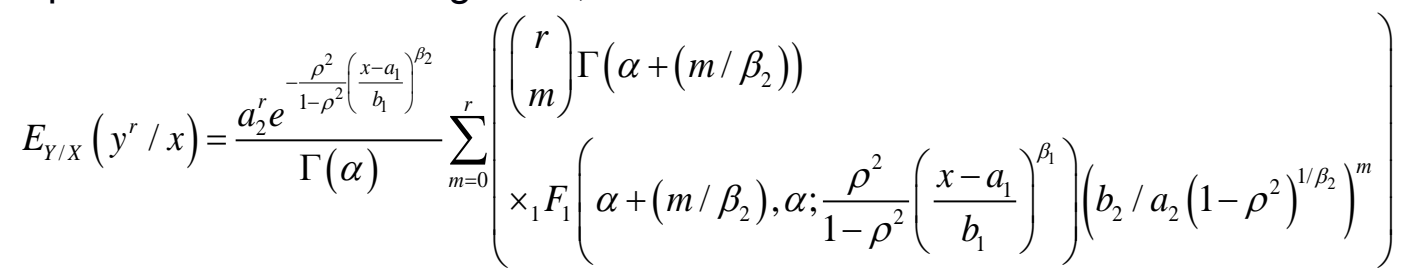

From (3.10), If $r=1,2,3,4$ then the following Conditional moments are given as

$$
\begin{aligned}
& E_{Y / X}(y / x)=\frac{a_{2} e^{-\frac{\lambda^{2}}{1-\lambda^{2}}\left(\frac{x-a_{1}}{b_{1}}\right)^{\beta_{2}}}}{\Gamma(\alpha)} \sum_{m=0}^{1}\left(\begin{array}{l}
\left(\begin{array}{c}
1 \\
m
\end{array}\right) \Gamma\left(\alpha+\left(m / \beta_{2}\right)\right) \\
\left.\times_{1} F_{1}\left(\alpha+\left(m / \beta_{2}\right), \alpha ; \frac{\lambda^{2}}{1-\lambda^{2}}\left(\frac{x-a_{1}}{b_{1}}\right)^{\beta_{1}}\right)\left(b_{2} / a_{2}\left(1-\lambda^{2}\right)^{1 / \beta_{2}}\right)^{m}\right)
\end{array}\right)
\end{aligned}
$$

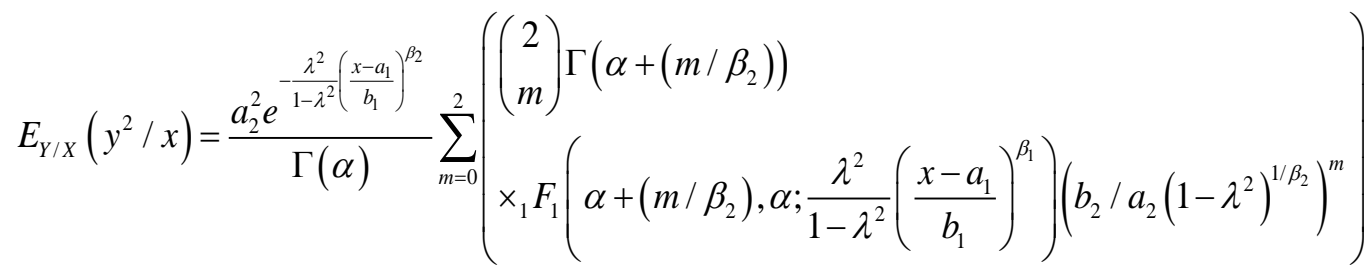

$$
\begin{aligned}
& E_{Y / X}\left(y^{3} / x\right)=\frac{a_{2}^{3} e^{-\frac{\lambda^{2}}{1-\lambda^{2}}\left(\frac{x-a_{1}}{b_{1}}\right)^{\beta_{2}}}}{\Gamma(\alpha)} \sum_{m=0}^{3}\left(\begin{array}{l}
\left(\begin{array}{c}
3 \\
m
\end{array}\right) \Gamma\left(\alpha+\left(m / \beta_{2}\right)\right) \\
\left.\times_{1} F_{1}\left(\alpha+\left(m / \beta_{2}\right), \alpha ; \frac{\lambda^{2}}{1-\lambda^{2}}\left(\frac{x-a_{1}}{b_{1}}\right)^{\beta_{1}}\right)\left(b_{2} / a_{2}\left(1-\lambda^{2}\right)^{1 / \beta_{2}}\right)^{m}\right)
\end{array}\right. \\
& E_{Y / X}\left(y^{4} / x\right)=\frac{a_{2}^{4} e^{-\frac{\lambda^{2}}{1-\lambda^{2}}\left(\frac{x-a_{1}}{b_{1}}\right)^{\beta_{2}}}}{\Gamma(\alpha)} \sum_{m=0}^{4}\left(\begin{array}{l}
\left(\begin{array}{c}
4 \\
m
\end{array}\right) \Gamma\left(\alpha+\left(m / \beta_{2}\right)\right) \\
\left.\times_{1} F_{1}\left(\alpha+\left(m / \beta_{2}\right), \alpha ; \frac{\lambda^{2}}{1-\lambda^{2}}\left(\frac{x-a_{1}}{b_{1}}\right)^{\beta_{1}}\right)\left(b_{2} / a_{2}\left(1-\lambda^{2}\right)^{1 / \beta_{2}}\right)^{m}\right)
\end{array}\right.
\end{aligned}
$$

Theorem 3.3: If $X$ and $Y$ are jointly distributed according to (1.1) then the product moment is 
$E_{X Y}\left(x^{m} y^{n}\right)=\frac{a_{1}^{m} a_{2}^{n}\left(1-\lambda^{2}\right)^{\alpha}}{\Gamma^{2}(\alpha)} \sum_{p=0}^{m} \sum_{q=0}^{n}\left(\begin{array}{l}{ }_{2} F_{1}\left(\alpha+\left(p / \beta_{1}\right), \alpha+\left(q / \beta_{2}\right), \alpha ; \lambda^{2}\right)\left(\begin{array}{l}m \\ p\end{array}\right)\left(\begin{array}{l}n \\ q\end{array}\right) \\ \left(b_{1}\left(1-\lambda^{2}\right)^{1 / \beta_{1}} / a_{1}\right)^{p}\left(b_{2}\left(1-\lambda^{2}\right)^{1 / \beta_{2}} / a_{2}\right)^{q} \\ \Gamma\left(\alpha+\left(p / \beta_{1}\right)\right) \Gamma\left(\alpha+\left(q / \beta_{2}\right)\right)\end{array}\right), p \neq q$

Where ${ }_{2} F_{1}()$ is the Gauss hyper geometric function.

Proof: The results follow on writing

$$
\begin{aligned}
& E\left(x^{m} y^{n}\right)=\frac{\left|\beta_{1}\right|\left|\beta_{2}\right|}{b_{1} b_{2} \Gamma^{2}(\alpha)\left(1-\lambda^{2}\right)^{\alpha}} \int_{a_{1} a_{2}}^{\infty} x^{m} y^{n}\left(\begin{array}{l}
\left(\frac{x-a_{1}}{b_{1}}\right)^{\alpha \beta_{1}-1}\left(\frac{y-a_{2}}{b_{2}}\right)^{\alpha \beta_{2}-1} e^{\left.-\frac{1}{1-\lambda^{2}}\left(\frac{x-a_{1}}{b_{1}}\right)^{\beta_{1}}+\left(\frac{y-a_{2}}{b_{2}}\right)^{\beta_{2}}\right)} \\
\times_{0} F_{1}\left(\alpha ; \frac{\lambda^{2}}{\left(1-\lambda^{2}\right)^{2}}\left(\frac{x-a_{1}}{b_{1}}\right)^{\beta_{1}}\left(\frac{y-a_{2}}{b_{2}}\right)^{\beta_{2}}\right)
\end{array}\right) d x d y \\
& E_{X Y}\left(x^{m} y^{n}\right)=\frac{a_{1}^{m} a_{2}^{n}\left(1-\lambda^{2}\right)^{\alpha}}{\Gamma^{2}(\alpha)} \sum_{p=0}^{m} \sum_{q=0}^{n}\left(\begin{array}{l}
{ }_{2} F_{1}\left(\alpha+\left(p / \beta_{1}\right), \alpha+\left(q / \beta_{2}\right), \alpha ; \lambda^{2}\right)\left(\begin{array}{l}
m \\
p
\end{array}\right)\left(\begin{array}{l}
n \\
q
\end{array}\right) \\
\left(b_{1}\left(1-\lambda^{2}\right)^{1 / \beta_{1}} / a_{1}\right)^{p}\left(b_{2}\left(1-\lambda^{2}\right)^{1 / \beta_{2}} / a_{2}\right)^{q} \\
\Gamma\left(\alpha+\left(p / \beta_{1}\right)\right) \Gamma\left(\alpha+\left(q / \beta_{2}\right)\right)
\end{array}\right), p \neq q
\end{aligned}
$$

Corollary-1. If $X$ and $Y$ are jointly distributed according to (1.1) then the first order Product moment, Co-variance and Correlation Co-efficient between two Amoroso variables are given as

$$
\begin{aligned}
& E_{X Y}(x y)=\left(\begin{array}{l}
a_{1} a_{2}\left(1-\rho^{2}\right)_{1} F_{0}\left(\alpha ; \lambda^{2}\right)+\left(b_{1} a_{2}\right)\left(1-\lambda^{2}\right)^{\left(1 / \beta_{1}\right)+1}{ }_{1} F_{0}\left(\alpha+\left(1 / \beta_{1}\right) ; \lambda^{2}\right) \\
+\left(b_{2} a_{1}\right)\left(1-\lambda^{2}\right)^{\left(1 / \beta_{2}\right)+1}{ }_{1} F_{0}\left(\alpha+\left(1 / \beta_{2}\right) ; \lambda^{2}\right) \\
+\left(\begin{array}{l}
\frac{b_{1} b_{2}}{\Gamma^{2}(\alpha)} \Gamma\left(\alpha+\left(1 / \beta_{1}\right)\right) \Gamma\left(\alpha+\left(1 / \beta_{1}\right)\right)\left(1-\lambda^{2}\right)^{\left(1 / \beta_{1}\right)+\left(1 / \beta_{2}\right)+1} \\
\times_{2} F_{1}\left(\alpha+\left(1 / \beta_{1}\right), \alpha+\left(1 / \beta_{2}\right), \alpha ; \lambda^{2}\right)
\end{array}\right)
\end{array}\right) \\
& \operatorname{COV}_{X, Y}(x, y)=\left(\begin{array}{l}
a_{1} a_{2}\left(\left(1-\lambda^{2}\right)_{1} F_{0}\left(\alpha ; \lambda^{2}\right)-1\right) \\
+\left(a_{1} b_{2}\right)\left(\left(1-\lambda^{2}\right)^{\left(1 / \beta_{2}\right)+1}{ }_{1} F_{0}\left(\alpha+\left(1 / \beta_{2}\right) ; \lambda^{2}\right)-\frac{\Gamma\left(\alpha+\left(1 / \beta_{2}\right)\right)}{\Gamma(\alpha)}\right) \\
+\left(a_{2} b_{1}\right)\left(\left(1-\lambda^{2}\right)^{\left(1 / \beta_{1}\right)+1}{ }_{1} F_{0}\left(\alpha+\left(1 / \beta_{1}\right) ; \lambda^{2}\right)-\frac{\Gamma\left(\alpha+\left(1 / \beta_{1}\right)\right)}{\Gamma(\alpha)}\right) \\
+\frac{b_{1} b_{2}}{\Gamma^{2}(\alpha)} \Gamma\left(\alpha+\left(1 / \beta_{1}\right)\right) \Gamma\left(\alpha+\left(1 / \beta_{1}\right)\right)\left(\begin{array}{l}
\left(\left(1-\lambda^{2}\right)^{\left(1 / \beta_{1}\right)+\left(1 / \beta_{2}\right)+1}\right) \\
{ }_{2} F_{1}\left(\alpha+\left(1 / \beta_{1}\right), \alpha+\left(1 / \beta_{2}\right), \alpha ; \lambda^{2}\right)-1
\end{array}\right)
\end{array}\right)
\end{aligned}
$$


$\rho_{X, Y}(x, y)=\left(\frac{\Gamma(\alpha)}{b_{1} b_{2}}\right) \frac{\left(\begin{array}{l}a_{1} a_{2}\left(\left(1-\lambda^{2}\right)_{1} F_{0}\left(\alpha ; \lambda^{2}\right)-1\right)+\left(a_{1} b_{2}\right)\left(\left(1-\lambda^{2}\right)^{\left(1 / \beta_{2}\right)+1}{ }_{1} F_{0}\left(\alpha+\left(1 / \beta_{2}\right) ; \lambda^{2}\right)-\frac{\Gamma\left(\alpha+\left(1 / \beta_{2}\right)\right)}{\Gamma(\alpha)}\right) \\ +\left(a_{2} b_{1}\right)\left(\left(1-\lambda^{2}\right)^{\left(1 / \beta_{1}\right)+1}{ }_{1} F_{0}\left(\alpha+\left(1 / \beta_{1}\right) ; \lambda^{2}\right)-\frac{\Gamma\left(\alpha+\left(1 / \beta_{1}\right)\right)}{\Gamma(\alpha)}\right) \\ +\frac{b_{1} b_{2}}{\Gamma^{2}(\alpha)} \Gamma\left(\alpha+\left(1 / \beta_{1}\right)\right) \Gamma\left(\alpha+\left(1 / \beta_{1}\right)\right)\left(\begin{array}{l}\left(\left(1-\lambda^{2}\right)^{\left(1 / \beta_{1}\right)+\left(1 / \beta_{2}\right)+1}\right) \\ { }_{2} F_{1}\left(\alpha+\left(1 / \beta_{1}\right), \alpha+\left(1 / \beta_{2}\right), \alpha ; \lambda^{2}\right)-1\end{array}\right)\end{array}\right)}{\sqrt{\left(\Gamma\left(\alpha+\left(2 / \beta_{1}\right)\right)-\frac{\Gamma^{2}\left(\alpha+\left(1 / \beta_{1}\right)\right)}{\Gamma(\alpha)}\right) \times\left(\Gamma\left(\alpha+\left(2 / \beta_{2}\right)\right)-\frac{\Gamma^{2}\left(\alpha+\left(1 / \beta_{2}\right)\right)}{\Gamma(\alpha)}\right)}}$

Where $-1 \leq \rho_{X, Y}(x, y) \leq+1$ and ${ }_{1} F_{0}(),{ }_{2} F_{1}(\quad)$ are the hyper-geometric and Gauss hyper geometric function respectively.

Theorem 3.4: The Joint Shannon's differential entropy of the bivariate Amoroso distribution is

$$
h=\sum_{j=1}^{5} \omega_{j}\left(b_{1}, b_{2}, \alpha, \beta_{1}, \beta_{2}, \lambda\right)
$$

where

$$
\begin{aligned}
& \omega_{1}\left(b_{1}, b_{2}, \alpha, \beta_{1}, \beta_{2}, \lambda\right)=-\log \left(\frac{\left|\beta_{1}\right|\left|\beta_{2}\right|}{b_{1} b_{2} \Gamma^{2}(\alpha)\left(1-\lambda^{2}\right)^{\alpha}}\right) \\
& \omega_{2}\left(b_{1}, b_{2}, \alpha, \beta_{1}, \beta_{2}, \lambda\right)=-\frac{\left(\alpha \beta_{1}-1\right)\left(1-\lambda^{2}\right)^{\alpha}}{\beta_{1}}\left(\log \left(1-\lambda^{2}\right)_{1} F_{0}\left(\alpha ; \lambda^{2}\right)+\sum_{k=0}^{\infty} \frac{(\alpha)_{k} \Psi(\alpha+k)}{k !}\left(\lambda^{2}\right)^{k}\right) \\
& \omega_{3}\left(b_{1}, b_{2}, \alpha, \beta_{1}, \beta_{2}, \lambda\right)=-\frac{\left(\alpha \beta_{2}-1\right)\left(1-\lambda^{2}\right)^{\alpha}}{\beta_{2}}\left(\log \left(1-\lambda^{2}\right)_{1} F_{0}\left(\alpha ; \lambda^{2}\right)+\sum_{k=0}^{\infty} \frac{(\alpha)_{k} \Psi(\alpha+k)}{k !}\left(\lambda^{2}\right)^{k}\right)- \\
& \omega_{4}\left(b_{1}, b_{2}, \alpha, \beta_{1}, \beta_{2}, \lambda\right)=2 \alpha\left(1-\lambda^{2}\right)^{\alpha}{ }_{1} F_{0}\left(\alpha+1 ; \lambda^{2}\right) \\
& \left.\omega_{5}\left(b_{1}, b_{2}, \alpha, \beta_{1}, \beta_{2}, \lambda\right)=-\frac{\left|\beta_{1}\right|\left|\beta_{2}\right|}{b_{1} b_{2} \Gamma^{2}(\alpha)\left(1-\lambda^{2}\right)^{\alpha}} \int_{a_{1} a_{2}}^{\infty} \int_{0}^{\infty} \log \left(F_{0} F_{1}\left(\alpha ; \frac{\lambda^{2}}{\left(1-\lambda^{2}\right)^{2}}\left(\frac{x-a_{1}}{b_{1}}\right)^{\beta_{1}}\left(\frac{y-a_{2}}{b_{2}}\right)^{\beta_{2}}\right)\right)\left(\frac{x-a_{1}}{b_{1}}\right)^{\alpha \beta_{1}-1}\left(\frac{y-a_{2}}{b_{2}}\right)^{\alpha \beta_{2}-1}\right) d x d y
\end{aligned}
$$

and ${ }_{1} F_{0}(), \Psi()$ are the hyper geometric and di-gamma function respectively. Proof: It is found from

$$
h=-\int_{a_{1}}^{\infty} \int_{a_{2}}^{\infty} \log \left(f_{X, Y}(x, y)\right) f_{X, Y}(x, y) d x d y
$$




$$
\begin{gathered}
=\int_{a_{1} a_{2}}^{\infty} \int\left(\begin{array}{l}
-\log \left(\frac{\left|\beta_{1}\right|\left|\beta_{2}\right|}{b_{1} b_{2} \Gamma^{2}(\alpha)\left(1-\lambda^{2}\right)^{\alpha}}\right)-\left(\alpha \beta_{1}-1\right) \log \left(\frac{x-a_{1}}{b_{1}}\right)-\left(\alpha \beta_{2}-1\right) \log \left(\frac{y-a_{2}}{b_{2}}\right) \\
\left.+\left(\frac{1}{1-\lambda^{2}}\left(\left(\frac{x-a_{1}}{b_{1}}\right)^{\beta_{1}}+\left(\frac{y-a_{2}}{b_{2}}\right)^{\beta_{2}}\right)\right)-\log \left(F_{0}\left(\alpha ; \frac{\lambda^{2}}{\left(1-\lambda^{2}\right)^{2}}\left(\frac{x-a_{1}}{b_{1}}\right)^{\beta_{1}}\left(\frac{y-a_{2}}{b_{2}}\right)^{\beta_{2}}\right)\right)\right) \\
x \frac{\left|\beta_{1}\right|\left|\beta_{2}\right|}{b_{1} b_{2} \Gamma^{2}(\alpha)\left(1-\lambda^{2}\right)^{\alpha}}\left(\frac{x-a_{1}}{b_{1}}\right)^{\alpha \beta_{1}-1}\left(\frac{y-a_{2}}{b_{2}}\right)^{\alpha \beta_{2}-1} e^{-\frac{1}{1-\lambda^{2}(}\left(\frac{x-a_{1}}{b_{1}}\right)^{\beta_{1}}+\left(\frac{y-a_{2}}{b_{2}}\right)^{\beta_{2}}}{ }_{0} F_{1}\left(\alpha ; \frac{\lambda^{2}}{\left(1-\lambda^{2}\right)^{2}}\left(\frac{x-a_{1}}{b_{1}}\right)^{\beta_{1}}\left(\frac{y-a_{2}}{b_{2}}\right)^{\beta_{2}}\right)
\end{array}\right) d x d y \\
h=\sum_{j=1}^{5} \omega_{j}\left(b_{1}, b_{2}, \alpha, \beta_{1}, \beta_{2}, \lambda\right)
\end{gathered}
$$

From (3.25), for the auxiliary function $\omega_{5}\left(b_{1}, b_{2}, \alpha, \beta_{1}, \beta_{2}, \lambda\right)$, it is not possible to derive an explicit expression to the integral, due to its non-convergence.

\section{Generating functions}

Theorem 4.1: The Moment generating function of the bivariate Amoroso distribution is

$$
M_{X, Y}\left(t_{1}, t_{2}\right)=\frac{\left(1-\lambda^{2}\right)^{\alpha} e^{a_{1} t_{1}+a_{2} t_{2}}}{\Gamma^{2}(\alpha)} \sum_{k=0}^{\infty}\left(\begin{array}{l}
\frac{\left(\lambda^{2}\right)^{k}}{(\alpha)_{k} k !}{ }_{1} \Psi_{0}\left[\left(\alpha+k, 1 / \beta_{1}\right) ; b_{1} t_{1}\left(1-\lambda^{2}\right)^{1 / \beta_{1}}\right] \\
\times_{1} \Psi_{0}\left[\left(\alpha+k, 1 / \beta_{2}\right) ; b_{2} t_{2}\left(1-\lambda^{2}\right)^{1 / \beta_{2}}\right]
\end{array}\right)-
$$

Where $\Gamma\left(\right.$ ) and ${ }_{1} \Psi_{0}(\quad)$ are the Gamma and Fox-Wright function respectively.

Proof: Let the moment generating function of a bivariate distribution is given as

$$
\left.\left.M_{X, Y}\left(t_{1}, t_{2}\right)=\frac{\left|\beta_{1}\right|\left|\beta_{2}\right|}{b_{1} b_{2} \Gamma^{2}(\alpha)\left(1-\lambda^{2}\right)^{\alpha}} \int_{a_{1} a_{2}}^{\infty} \int_{{ }_{0}}^{\infty} F_{1}\left(\alpha ; \frac{\lambda^{2}}{\left(1-\lambda^{2}\right)^{2}}\left(\frac{x-a_{1}}{b_{1}}\right)^{\beta_{1} x+t_{2} y}\left(\frac{x-a_{1}}{b_{1}}\right)^{\alpha \beta_{1}-1}\left(\frac{y-a_{2}}{b_{2}}\right)^{\beta_{2}}\right)^{\alpha \beta_{2}-1}-\frac{1}{1-\lambda^{2}}\left(\frac{x-a_{1}}{b_{1}}\right)^{\beta_{1}}+\left(\frac{y-a_{2}}{b_{2}}\right)^{\beta_{2}}\right)\right) d x d y
$$

By Setting $U=\left(\left(x-a_{1}\right) / b_{1}\right)^{\beta_{1}}, V=\left(\left(y-a_{2}\right) / b_{2}\right)^{\beta_{1}}$ and expand the exponential, hypergeometric function ${ }_{0} F_{1}()$, then the final integration result of $(4.2)$ is found to be

$$
M_{X, Y}\left(t_{1}, t_{2}\right)=\frac{\left(1-\lambda^{2}\right)^{\alpha} e^{a_{1} t_{1}+a_{2} t_{2}}}{\Gamma^{2}(\alpha)} \sum_{k=0}^{\infty}\left(\begin{array}{l}
\frac{\left(\lambda^{2}\right)^{k}}{(\alpha)_{k} k !}{ }^{k} \Psi_{0}\left[\left(\alpha+k, 1 / \beta_{1}\right) ; b_{1} t_{1}\left(1-\lambda^{2}\right)^{1 / \beta_{1}}\right] \\
\times_{1} \Psi_{0}\left[\left(\alpha+k, 1 / \beta_{2}\right) ; b_{2} t_{2}\left(1-\lambda^{2}\right)^{1 / \beta_{2}}\right]
\end{array}\right)
$$


Theorem 4.2: The Cumulant of the bivariate Amoroso distribution is $C_{X, Y}\left(t_{1}, t_{2}\right)=\alpha \log \left(1-\lambda^{2}\right)+a_{1} t_{1}+a_{2} t_{2}-2 \log \Gamma(\alpha)+\log \left(\sum_{k=0}^{\infty}\left(\begin{array}{l}\frac{\left(\lambda^{2}\right)^{k}}{(\alpha)_{k} k !} \Psi_{0}\left[\left(\alpha+k, 1 / \beta_{1}\right) ; b_{1} t_{1}\left(1-\lambda^{2}\right)^{1 / \beta_{1}}\right] \\ x_{1} \Psi_{0}\left[\left(\alpha+k, 1 / \beta_{2}\right) ; b_{2} t_{2}\left(1-\lambda^{2}\right)^{1 / \beta_{2}}\right]\end{array}\right)\right)$

Proof: It is found from $C_{X, Y}\left(t_{1}, t_{2}\right)=\log M_{X, Y}\left(t_{1}, t_{2}\right)$

Theorem 4.3: The Characteristic function of the bivariate Amoroso distribution is

$\phi_{X, Y}\left(t_{1}, t_{2}\right)=\frac{\left(1-\lambda^{2}\right)^{\alpha} e^{i\left(a_{1} t_{1}+a_{2} t_{2}\right)}}{\Gamma^{2}(\alpha)} \sum_{k=0}^{\infty}\left(\begin{array}{l}\frac{\left(\lambda^{2}\right)^{k}}{(\alpha)_{k} k !} \Psi_{0}\left[\left(\alpha+k, 1 / \beta_{1}\right) ; i b_{1} t_{1}\left(1-\lambda^{2}\right)^{1 / \beta_{1}}\right] \\ x_{1} \Psi_{0}\left[\left(\alpha+k, 1 / \beta_{2}\right) ; i b_{2} t_{2}\left(1-\lambda^{2}\right)^{1 / \beta_{2}}\right]\end{array}\right)$

Where $\Gamma\left(\right.$ ) and ${ }_{1} \Psi_{0}($ ) are the Gamma and Fox-Wright function with complex arguments respectively.

Proof: Let the Characteristic function of a bivariate distribution is given as

$$
\begin{aligned}
& \phi_{X, Y}\left(t_{1}, t_{2}\right)=\int_{a_{1}}^{\infty} \int_{a_{2}}^{\infty} e^{i\left(t_{1} x+t_{2} y\right)} f_{X, Y}(x, y) d x d y \\
& \left.=\frac{\left|\beta_{1}\right|\left|\beta_{2}\right|}{b_{1} b_{2} \Gamma^{2}(\alpha)\left(1-\lambda^{2}\right)^{\alpha}} \int_{a_{1} a_{2}}^{\infty} \int_{0}^{\infty} e_{0}^{i\left(t_{1} x+t_{2} y\right)}\left(\frac{x-a_{1}}{b_{1}}\right)^{\alpha \beta_{1}-1}\left(\frac{y-a_{2}}{b_{2}}\right)^{\alpha \beta_{2}-1} e^{-\frac{1}{1-\lambda^{2}}\left(\left(\frac{x-a_{1}}{b_{1}}\right)^{\beta_{1}}+\left(\frac{y-a_{2}}{b_{2}}\right)^{\beta_{2}}\right)}\right) d x d y
\end{aligned}
$$

For (4.5), it is evident from theorem 4.2, the integration is obvious and final expression Cf is given as

$$
\phi_{X, Y}\left(t_{1}, t_{2}\right)=\frac{\left(1-\lambda^{2}\right)^{\alpha} e^{i\left(a_{1} t_{1}+a_{2} t_{2}\right)}}{\Gamma^{2}(\alpha)} \sum_{k=0}^{\infty}\left(\begin{array}{l}
\frac{\left(\lambda^{2}\right)^{k}}{(\alpha)_{k} k !} \Psi_{0}\left[\left(\alpha+k, 1 / \beta_{1}\right) ; i b_{1} t_{1}\left(1-\lambda^{2}\right)^{1 / \beta_{1}}\right] \\
x_{1} \Psi_{0}\left[\left(\alpha+k, 1 / \beta_{2}\right) ; i b_{2} t_{2}\left(1-\lambda^{2}\right)^{1 / \beta_{2}}\right]
\end{array}\right)
$$

Theorem 4.4: The survival function of bivariate Amoroso distribution is $S_{X, Y}(x, y)=1-\frac{1}{\Gamma^{2}(\alpha)\left(1-\lambda^{2}\right)^{\alpha}} \sum_{k=0}^{\infty} \frac{1}{(\alpha)_{k} k !}\left(\frac{\lambda^{2 k}}{\left(1-\lambda^{2}\right)^{2 k}} \gamma\left(\alpha+k, \frac{\left(\left(x-a_{1}\right) / b_{1}\right)^{\beta_{1}}}{1-\lambda^{2}}\right) \gamma\left(\alpha+k, \frac{\left(\left(y-a_{2}\right) / b_{2}\right)^{\beta_{2}}}{1-\lambda^{2}}\right)\right)$

where $\gamma()$ is the lower incomplete Gamma function.

Proof: It is found from the following fact

$$
S_{X, Y}(x, y)=1-F_{X, Y}(x, y)
$$


Theorem 4.5: The hazard function of the bivariate Amoroso distribution is

$$
\begin{aligned}
& \frac{\left|\beta_{1}\right|\left|\beta_{2}\right|}{b_{1} b_{2} \Gamma^{2}(\alpha)\left(1-\lambda^{2}\right)^{\alpha}}\left(\left(\frac{x-a_{1}}{b_{1}}\right)^{\alpha \beta_{1}-1}\left(\frac{y-a_{2}}{b_{2}}\right)^{\alpha \beta_{2}-1} e^{\left.-\frac{1}{1-\lambda^{2}}\left(\frac{x-a_{1}}{b_{1}}\right)^{\beta_{1}}+\left(\frac{y-a_{2}}{b_{2}}\right)^{\beta_{2}}\right)}{ }_{0} F_{1}\left(\alpha ; \frac{\lambda^{2}}{\left(1-\lambda^{2}\right)^{2}}\left(\frac{x-a_{1}}{b_{1}}\right)^{\beta_{1}}\left(\frac{y-a_{2}}{b_{2}}\right)^{\beta_{2}}\right)\right)
\end{aligned}
$$

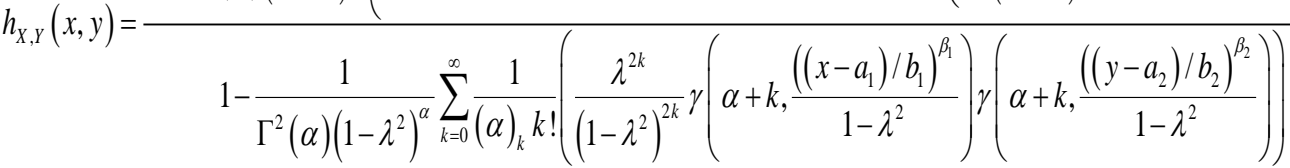

Proof: It is found from

$$
h_{X, Y}(x, y)=\frac{f_{X, Y}(x, y)}{S_{X, Y}(x, y)} \text { and } S_{X, Y}(x, y)=1-F_{X, Y}(x, y)
$$

Theorem 4.6: The Cumulative hazard function of the bivariate Amoroso distribution is

$$
H_{X, Y}(x, y)=-\log \left(1-\frac{1}{\Gamma^{2}(\alpha)\left(1-\lambda^{2}\right)^{\alpha}} \sum_{k=0}^{\infty} \frac{1}{(\alpha)_{k} k !}\left(\frac{\lambda^{2 k}}{\left(1-\lambda^{2}\right)^{2 k}} \gamma\left(\alpha+k, \frac{\left(\left(x-a_{1}\right) / b_{1}\right)^{\beta_{1}}}{1-\lambda^{2}}\right) \gamma\left(\alpha+k, \frac{\left(\left(y-a_{2}\right) / b_{2}\right)^{\beta_{2}}}{1-\lambda^{2}}\right)\right)\right)
$$

Proof: Let the Cumulative hazard function of a bivariate distribution is given as

$$
\begin{gathered}
H_{X, Y}(x, y)=-\log \left(1-F_{X, Y}(x, y)\right) \\
=-\log \left(S_{X, Y}(x, y)\right) \\
H_{X, Y}(x, y)=-\log \left(1-\frac{1}{\Gamma^{2}(\alpha)\left(1-\lambda^{2}\right)^{\alpha}} \sum_{k=0}^{\infty} \frac{1}{(\alpha)_{k} k !}\left(\frac{\lambda^{2 k}}{\left(1-\lambda^{2}\right)^{2 k}} \gamma\left(\alpha+k \frac{\left(\left(x-a_{1}\right) / b_{1}\right)^{\beta_{1}}}{1-\lambda^{2}}\right) \gamma\left(\alpha+k, \frac{\left(\left(y-a_{2}\right) / b_{2}\right)^{\beta_{2}}}{1-\lambda^{2}}\right)\right)\right)
\end{gathered}
$$

\section{Some Special Cases}

Result 5.1: From (1.1) and if the dependency co-efficient is zero $(l=0)$, then the density function of bivariate amoroso distribution is the product of two densities of univariate amoroso distributions.

Result 5.2 - Table in Appendix 1 shows the special cases of Bivariate Amoroso distribution from (1.1) for different settings of parameters are given.

Result 5.3: From the special cases of Result (5.2) by shifting the Location, Scale and Shape parameters we can easily derive $\left(\begin{array}{c}40 \\ 2\end{array}\right)=780$ mixtures of bivariate distributions.

Result 5.4: The probability surfaces of the special cases of bivariate standard Amoroso distribution for the selected values of parameters are visualized in Appendix 2.

\section{Parameter estimation}

Result 6.1: We consider the estimation by the method of maximum likelihood. The loglikelihood function for a random sample $x_{1}, x_{2}, x_{3}, \mathrm{~K}, x_{n-1}, x_{n}$ from (1.1) is 
$\log L\left(a_{1}, a_{2}, b_{1}, b_{2}, \alpha, \beta_{1}, \beta_{2}, \lambda\right)=\left(\begin{array}{l}n \log \left(\frac{\left|\beta_{1}\right|\left|\beta_{2}\right|}{b_{1} b_{2} \Gamma^{2}(\alpha)\left(1-\lambda^{2}\right)^{\alpha}}\right)+\left(\alpha \beta_{1}-1\right) \sum_{i=1}^{n} \log \left(\frac{x_{i}-a_{1}}{b_{1}}\right)+\left(\alpha \beta_{2}-1\right) \sum_{i=1}^{n} \log \left(\frac{y_{i}-a_{2}}{b_{2}}\right) \\ +\sum_{i=1}^{n} \log _{0} F_{1}\left(\alpha ; \frac{\lambda^{2}}{\left(1-\lambda^{2}\right)^{2}}\left(\frac{x_{i}-a_{1}}{b_{1}}\right)^{\beta_{1}}\left(\frac{y_{i}-a_{2}}{b_{2}}\right)^{\beta_{2}}\right)-\frac{1}{1-\lambda^{2}}\left(\sum_{i=1}^{n}\left(\frac{x_{i}-a_{1}}{b_{1}}\right)^{\beta_{1}}+\sum_{i=1}^{n}\left(\frac{y_{i}-a_{2}}{b_{2}}\right)^{\beta_{2}}\right)\end{array}\right)-$

The first order derivatives of (6.1) with respect to eight parameters are

$\frac{\partial \log L}{\partial a_{1}}=-\left(\alpha \beta_{1}-1\right) \sum_{i=1}^{n}\left(\frac{1}{x_{i}-a_{1}}\right)+\sum_{i=1}^{n}\left(\frac{{ }_{0} F_{1\left(a_{1}\right)}^{\prime}\left(\alpha ; \frac{\lambda^{2}}{\left(1-\lambda^{2}\right)^{2}}\left(\frac{x_{i}-a_{1}}{b_{1}}\right)^{\beta_{1}}\left(\frac{y_{i}-a_{2}}{b_{2}}\right)^{\beta_{2}}\right)}{{ }_{0} F_{1}\left(\alpha ; \frac{\lambda^{2}}{\left(1-\lambda^{2}\right)^{2}}\left(\frac{x_{i}-a_{1}}{b_{1}}\right)^{\beta_{1}}\left(\frac{y_{i}-a_{2}}{b_{2}}\right)^{\beta_{2}}\right)}\right)-\frac{1}{1-\lambda^{2}} \sum_{i=1}^{n}\left(\frac{1}{x_{i}-a_{1}}\right)$

$\frac{\partial \log L}{\partial a_{2}}=-\left(\alpha \beta_{2}-1\right) \sum_{i=1}^{n}\left(\frac{1}{y_{i}-a_{2}}\right)+\sum_{i=1}^{n}\left(\frac{{ }_{0} F_{1\left(a_{2}\right)}^{\prime}\left(\alpha ; \frac{\lambda^{2}}{\left(1-\lambda^{2}\right)^{2}}\left(\frac{x_{i}-a_{1}}{b_{1}}\right)^{\beta_{1}}\left(\frac{y_{i}-a_{2}}{b_{2}}\right)^{\beta_{2}}\right)}{{ }_{0} F_{1}\left(\alpha ; \frac{\lambda^{2}}{\left(1-\lambda^{2}\right)^{2}}\left(\frac{x_{i}-a_{1}}{b_{1}}\right)^{\beta_{1}}\left(\frac{y_{i}-a_{2}}{b_{2}}\right)^{\beta_{2}}\right)}\right)-\frac{1}{1-\lambda^{2}} \sum_{i=1}^{n}\left(\frac{1}{y_{i}-a_{2}}\right)-$

$\frac{\partial \log L}{\partial b_{1}}=-\frac{n}{b_{1}}-\frac{n\left(\alpha \beta_{1}-1\right)}{b_{1}}+\sum_{i=1}^{n}\left(\frac{{ }_{0} F_{1\left(b_{1}\right)}^{\prime}\left(\alpha ; \frac{\lambda^{2}}{\left(1-\lambda^{2}\right)^{2}}\left(\frac{x_{i}-a_{1}}{b_{1}}\right)^{\beta_{1}}\left(\frac{y_{i}-a_{2}}{b_{2}}\right)^{\beta_{2}}\right)}{{ }_{0} F_{1}\left(\alpha ; \frac{\lambda^{2}}{\left(1-\lambda^{2}\right)^{2}}\left(\frac{x_{i}-a_{1}}{b_{1}}\right)^{\beta_{1}}\left(\frac{y_{i}-a_{2}}{b_{2}}\right)^{\beta_{2}}\right)}\right)+\frac{\beta_{1}}{b_{1}\left(1-\lambda^{2}\right)} \sum_{i=1}^{n}\left(\frac{x_{i}-a_{1}}{b_{1}}\right)^{\beta_{1}}-$

$\frac{\partial \log L}{\partial b_{2}}=-\frac{n \alpha \beta_{2}}{b_{2}}+\sum_{i=1}^{n}\left(\frac{{ }_{0} F_{1\left(b_{1}\right)}^{\prime}\left(\alpha ; \frac{\lambda^{2}}{\left(1-\lambda^{2}\right)^{2}}\left(\frac{x_{i}-a_{1}}{b_{1}}\right)^{\beta_{1}}\left(\frac{y_{i}-a_{2}}{b_{2}}\right)^{\beta_{2}}\right)}{{ }_{0} F_{1}\left(\alpha ; \frac{\lambda^{2}}{\left(1-\lambda^{2}\right)^{2}}\left(\frac{x_{i}-a_{1}}{b_{1}}\right)^{\beta_{1}}\left(\frac{y_{i}-a_{2}}{b_{2}}\right)^{\beta_{2}}\right)}\right)+\frac{\beta_{2}}{b_{2}\left(1-\lambda^{2}\right)} \sum_{i=1}^{n}\left(\frac{y_{i}-a_{1}}{b_{1}}\right)^{\beta_{2}}-$

$\frac{\partial \log L}{\partial \alpha}=\left(\begin{array}{l}-2 n \Psi(\alpha)-n \log \left(1-\lambda^{2}\right)+\beta_{1} \sum_{i=1}^{n} \log \left(\frac{x_{i}-a_{1}}{b_{1}}\right)+\beta_{2} \sum_{i=1}^{n} \log \left(\frac{y_{i}-a_{2}}{b_{2}}\right) \\ +\sum_{i=1}^{n}\left(\frac{{ }_{0} F_{1(\alpha)}^{\prime}\left(\alpha ; \frac{\lambda^{2}}{\left(1-\lambda^{2}\right)^{2}}\left(\frac{x_{i}-a_{1}}{b_{1}}\right)^{\beta_{1}}\left(\frac{y_{i}-a_{2}}{b_{2}}\right)^{\beta_{2}}\right)}{{ }_{0} F_{1}\left(\alpha ; \frac{\lambda^{2}}{\left(1-\lambda^{2}\right)^{2}}\left(\frac{x_{i}-a_{1}}{b_{1}}\right)^{\beta_{1}}\left(\frac{y_{i}-a_{2}}{b_{2}}\right)^{\beta_{2}}\right)}\right)\end{array}\right)$ 


$$
\frac{\partial \log L}{\partial \beta_{1}}=\left(\begin{array}{l}
\left.\frac{n\left(a b s\left(1, \beta_{1}\right)\right)}{\left|\beta_{1}\right|}+\alpha \sum_{i=1}^{n} \log \left(\frac{x_{i}-a_{1}}{b_{1}}\right)+\sum_{i=1}^{n}\left(\frac{{ }_{0} F_{1\left(\beta_{1}\right)}^{\prime}\left(\alpha ; \frac{\lambda^{2}}{\left(1-\lambda^{2}\right)^{2}}\left(\frac{x_{i}-a_{1}}{b_{1}}\right)^{\beta_{1}}\left(\frac{y_{i}-a_{2}}{b_{2}}\right)^{\beta_{2}}\right)}{{ }_{0} F_{1}\left(\alpha ; \frac{\lambda^{2}}{\left(1-\lambda^{2}\right)^{2}}\left(\frac{x_{i}-a_{1}}{b_{1}}\right)^{\beta_{1}}\left(\frac{y_{i}-a_{2}}{b_{2}}\right)^{\beta_{2}}\right)}\right)\right) \\
\frac{\partial \log L}{\partial \beta_{2}}=\left(\begin{array}{l}
\frac{n\left(a b s\left(1, \beta_{2}\right)\right)}{\left|\beta_{2}\right|}+\alpha \sum_{i=1}^{n}\left(\left(\frac{x_{i}-a_{1}}{b_{1}}\right)^{\beta_{1}} \log \left(\frac{y_{i}-a_{2}}{b_{2}}\right)+\sum_{i=1}^{n}\left(\frac{x_{i}-a_{1}}{b_{1}}\right)\right) \\
-\frac{1}{1-\lambda^{2}} \sum_{i=1}^{n}\left(\left(\frac{y_{i}-a_{2}}{b_{2}}\right)^{\beta_{2}} \log \left(\alpha ; \frac{\lambda^{2}}{\left(1-\lambda^{2}\right)^{2}}\left(\frac{x_{i}-a_{1}}{b_{1}}\right)^{\beta_{1}}\left(\frac{y_{i}-a_{2}}{b_{2}}\right)^{\beta_{2}}\right)\right. \\
{ }_{0} F_{1}\left(\alpha ; \frac{\lambda^{2}}{\left(1-\lambda^{2}\right)^{2}}\left(\frac{x_{i}-a_{1}}{b_{1}}\right)^{\beta_{1}}\left(\frac{y_{i}-a_{2}}{b_{2}}\right)^{\beta_{2}}\right)
\end{array}\right)
\end{array}\right)
$$

and

$$
\frac{\partial \log L}{\partial \lambda}=\frac{2 \lambda}{1-\lambda^{2}}\left(n \alpha+\sum_{i=1}^{n}\left(\frac{{ }_{0} F_{1(\lambda)}^{\prime}\left(\alpha ; \frac{\lambda^{2}}{\left(1-\lambda^{2}\right)^{2}}\left(\frac{x_{i}-a_{1}}{b_{1}}\right)^{\beta_{1}}\left(\frac{y_{i}-a_{2}}{b_{2}}\right)^{\beta_{2}}\right)}{{ }_{0} F_{1}\left(\alpha ; \frac{\lambda^{2}}{\left(1-\lambda^{2}\right)^{2}}\left(\frac{x_{i}-a_{1}}{b_{1}}\right)^{\beta_{1}}\left(\frac{y_{i}-a_{2}}{b_{2}}\right)^{\beta_{2}}\right)}\right)-\frac{1}{1-\lambda^{2}}\left(\sum_{i=1}^{n}\left(\frac{x_{i}-a_{1}}{b_{1}}\right)^{\beta_{1}}+\sum_{i=1}^{n}\left(\frac{y_{i}-a_{2}}{b_{2}}\right)^{\beta_{2}}\right)\right)-
$$

Setting these expressions to zero and solving them simultaneously yields the maximum likelihood estimates of the eight parameters.

Result 6.2: As a second approach, the authors realized that the computational complexity of the maximum likelihood estimation of the proposed distribution is Painstaking due to the high non-linearity of parameters and the involvement of special functions. Hence they moved to the Non-linear programming approach by adopting Constrained Maximum likelihood method to estimate the parameters of the Bivariate Amoroso distribution and the idea is to Maximizing the log-likelihood function from (6.1) under some restriction and parameter constraints and it given as follows: 


$$
\operatorname{Max}\left(\log L\left(a_{1}, a_{2}, b_{1}, b_{2}, \alpha, \beta_{1}, \beta_{2}, \lambda\right)\right)=\left(\begin{array}{l}
n \log \left(\frac{\left|\beta_{1}\right|\left|\beta_{2}\right|}{b_{1} b_{2} \Gamma^{2}(\alpha)\left(1-\lambda^{2}\right)^{\alpha}}\right)+\left(\alpha \beta_{1}-1\right) \sum_{i=1}^{n} \log \left(\frac{x_{i}-a_{1}}{b_{1}}\right)+\left(\alpha \beta_{2}-1\right) \sum_{i=1}^{n} \log \left(\frac{y_{i}-a_{2}}{b_{2}}\right) \\
+\sum_{i=1}^{n} \log _{0} F_{1}\left(\alpha ; \frac{\lambda^{2}}{\left(1-\lambda^{2}\right)^{2}}\left(\frac{x_{i}-a_{1}}{b_{1}}\right)^{\beta_{1}}\left(\frac{y_{i}-a_{2}}{b_{2}}\right)^{\beta_{2}}\right)-\frac{1}{1-\lambda^{2}}\left(\sum_{i=1}^{n}\left(\frac{x_{i}-a_{1}}{b_{1}}\right)^{\beta_{1}}+\sum_{i=1}^{n}\left(\frac{y_{i}-a_{2}}{b_{2}}\right)^{\beta_{2}}\right)
\end{array}\right)-
$$

Subject to the constraints

\section{Application}

$$
\left(\begin{array}{c}
n \log \left(\frac{\left|\beta_{1}\right|\left|\beta_{2}\right|}{b_{1} b_{2} \Gamma^{2}(\alpha)\left(1-\lambda^{2}\right)^{\alpha}}\right)+\left(\alpha \beta_{1}-1\right) \sum_{i=1}^{n} \log \left(\frac{x_{i}-a_{1}}{b_{1}}\right)+\left(\alpha \beta_{2}-1\right) \sum_{i=1}^{n} \log \left(\frac{y_{i}-a_{2}}{b_{2}}\right) \\
+\sum_{i=1}^{n} \log _{0} F_{1}\left(\alpha ; \frac{\lambda^{2}}{\left(1-\lambda^{2}\right)^{2}}\left(\frac{x_{i}-a_{1}}{b_{1}}\right)^{\beta_{1}}\left(\frac{y_{i}-a_{2}}{b_{2}}\right)^{\beta_{2}}\right)-\frac{1}{1-\lambda^{2}}\left(\sum_{i=1}^{n}\left(\frac{x_{i}-a_{1}}{b_{1}}\right)^{\beta_{1}}+\sum_{i=1}^{n}\left(\frac{y_{i}-a_{2}}{b_{2}}\right)^{\beta_{2}}\right) \\
a_{1}, a_{2}, \mathrm{~b}_{1}, \mathrm{~b}_{2} \alpha, \beta_{1}, \beta_{2} \geq 0 \\
-1 \leq \lambda \leq+1
\end{array}\right.
$$

In this Section, we now illustrate an application of fitting the Bivariate Amoroso distribution for the 4 variables namely Sepal length $\left(X_{1}\right)$, Sepal Width $\left(X_{2}\right)$, Petal length $\left(X_{3}\right)$, petal width $\left(X_{4}\right)$ (Plants of Iris Setosa, Versicolour, Virginica in $\mathrm{cms}$ ) with a random sample size of $(n=30)$. The variables are collected from the Database namely Iris Plants data(1936) and the information regarding the databases are clearly given in the references. The authors realized the computational difficulties in the classical unconstrained Maximum likelihood method and hence they adopted Non-linear programming approach to estimate the parameters by using the constrained Maximum likelihood method with the help of Optimization Module in the best known Mathematical Software Maple version 18 and the estimated results of Parameters with a decimal approximation are tabulated in Table 1 and the fitted Probability Surface are visualized in Figure 1.
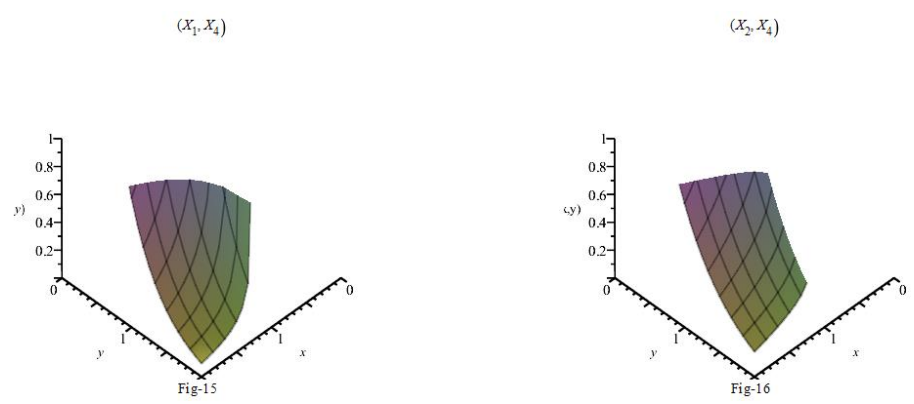

Figure 1: Fitted Probability Surface of the Bivariate Amoroso distribution 
Table 1: Parameter estimates of Bivariate Amoroso distribution.

\begin{tabular}{|c|c|c|c|c|c|c|c|c|c|}
\hline \multirow{4}{*}{$\begin{array}{l}\text { Bivariate } \\
\text { Variables }\end{array}$} & \multicolumn{9}{|c|}{ Constrained Maximum Likelihood estimates } \\
\hline & & & & & ran & ters & & & Maximized \\
\hline & \multicolumn{2}{|c|}{ Location } & \multicolumn{2}{|c|}{ Scale } & \multicolumn{3}{|c|}{ Shape } & \multirow{2}{*}{$\frac{\mathrm{DE}}{\stackrel{\$}{\mathrm{~d}}}$} & \multirow{2}{*}{$\begin{array}{l}\text { Log- } \\
\text { likelihood }\end{array}$} \\
\hline & $\mu_{1}$ & $\mu$ & $\mathbb{B}_{1}^{\mu}$ & $\mu_{2}$ & $d \mathrm{~d}$ & $\beta_{1}^{\mathbf{L}}$ & $\Phi_{2}$ & & \\
\hline${ }^{*} X_{1}, X_{2}$ & - & - & - & - & - & - & - & - & - \\
\hline${ }^{*} X_{1}, X_{3}$ & - & - & - & - & - & - & - & - & 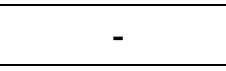 \\
\hline$X_{1}, X_{4}$ & 1.2341 & 1.2651 & 1.6535 & 0.4009 & 1 & 0.49473 & 1 & 0 & -0.000316 \\
\hline${ }^{*} X_{2}, X_{3}$ & - & - & - & - & - & - & - & - & - \\
\hline$X_{2}, X_{4}$ & 1.2177 & 1.2156 & 1.3171 & 0.62520 & 1 & 0.92535 & 1 & 0 & -0.0006277 \\
\hline${ }^{*} X_{3}, X_{4}$ & - & - & - & - & - & - & - & - & - \\
\hline
\end{tabular}

* Optimal Solutions not attained DE- Dependency Co-efficient

\section{Conclusion}

This paper proposed a bivariate extension of the univariate amoroso distribution and the characteristics of the bivariate distribution are extensively studied. Usually, several univariate distributions are extended to the bivariate case and the extension of generalized distributions to the bivariate cases are rarely touched. Moreover, the maximum likelihood estimation of the parameters of bivariate amoroso distribution is also discussed and the computational complexity of the parameters may reduce the practicality and application of this distribution. Finally, it's multivariate generalization leads to arise different family of generalized distributions and the authors left the computational complexity of parameters in the unconstrained maximum likelihood estimation of the proposed distribution for future work.

\section{References}

Abd-Elfattah, A., Fergany, H., \& Omima, A. (2010). Goodness of fit tests for generalized Fréchet distribution. Australian Journal of Basic and Applied Sciences, 4(2): 286-301.

Amoroso, F., \& Mignotte, M. (1996). On the distribution on the roots of polynomials. Annales de l'institut Fourier, 46(5), 1275-1291.

Bhatti, F. A., Ali, A., Hamedani, G., \& Ahmad, M. (2019). Pak. J. Statist. 2019 Vol. 35 (1), 25-51 ON THE GENERALIZED LOG BURR III DISTRIBUTION: DEVELOPMENT, PROPERTIES, CHARACTERIZATIONS AND APPLICATIONS. Pak. J. Statist, 35(1): $25-51$.

Clementi, F., Di Matteo, T., Gallegati, M., \& Kaniadakis, G. (2008). The K-generalized distribution: A new descriptive model for the size distribution of incomes. Physica A: Statistical Mechanics and lts Applications, 387(13): 3201-3208.

Cordeiro, G. M., Pescim, R. R., \& Ortega, E. M. (2012). The Kumaraswamy generalized half-normal distribution for skewed positive data. Journal of Data Science, 10(2): 195224. 
Gradshteyn, I. S., \& Ryzhik, I. M. (2014). Table of integrals, series, and products. San Diego (US): Academic press.

Hamedani, G. (2011). Characterizations of the Shakil-Kibria-Singh Distribution. Austrian Journal of Statistics, 40(3): 201-207.

Mansoor, M., Tahir, M., Cordeiro, G. M., Provost, S. B., \& Alzaatreh, A. (2019). The Marshall-Olkin logistic-exponential distribution. Communications in Statistics-Theory and Methods, 48(2): 220-234.

Merovci, F. (2014). Transmuted generalized Rayleigh distribution. Journal of Statistics Applications \& Probability, 3(1): 9-20.

Moghaddam, M. D., Mills, J., \& Serota, R. (2019). Generalized beta prime distribution: Stochastic model of economic exchange and properties of inequality indices. ArXiv Preprint ArXiv:1906.04822, 1-19.

Nassar, M., \& Nada, N. (2011). The beta generalized Pareto distribution. Journal of Statistics: Advances in Theory and Applications, 6(1/2): 1-17.

Oladipo, A. T. (2019). Bounds for Probabilities of the Generalized Distribution Defined by Generalized Polylogarithm. Journal of Mathematics (ISSN 1016-2526), 51(7): 19-26.

Potdar, K., \& Shirke, D. (2013). Inference for the parameters of generalized inverted family of distributions. ProbStat Forum, 6, 18-28.

Progri, I. F. (2016). Exponential generalized Beta distribution. J. Geol. Geoinfo. Geointel, 2016: 35-52.

Prudnikov, A. P., Brychkov, Y. A., \& Marichev, O. I. (1986). Integrals and series: special functions (Vol. 1, 2, and 3). Amsterdam (NL): Gordon and Breach Science Publishers.

Ramos, P. L., Achcar, J. A., Moala, F. A., Ramos, E., \& Louzada, F. (2017). Bayesian analysis of the generalized gamma distribution using non-informative priors. Statistics, 51(4): 824-843.

Ramos, P. L., \& Louzada, F. (2018). Bayesian reference analysis for the generalized gamma distribution. IEEE Communications Letters, 22(9): 1950-1953.

Scott, D. J., Würtz, D., Dong, C., \& Tran, T. T. (2011). Moments of the generalized hyperbolic distribution. Computational Statistics, 26(3): 459-476.

Tripathi, H., Yadav, A. S., Saha, M., \& Kumar, S. (2018). Generalized inverse xgamma distribution: A non-monotone hazard rate model. ArXiv Preprint ArXiv:1812.04933, 19.

VedoVatto, T., Nascimento, A., Lima, M., Pinho, L., Cordeiro, G., \& others. (2016). Some computational and theoretical aspects of the exponentiated generalized NadarajahHaghighi distribution. ArXiv Preprint ArXiv:1610.08876, 1-24. 


\section{APPENDIX 1}

Table 2: Special cases of Bivariate Amoroso distribution.

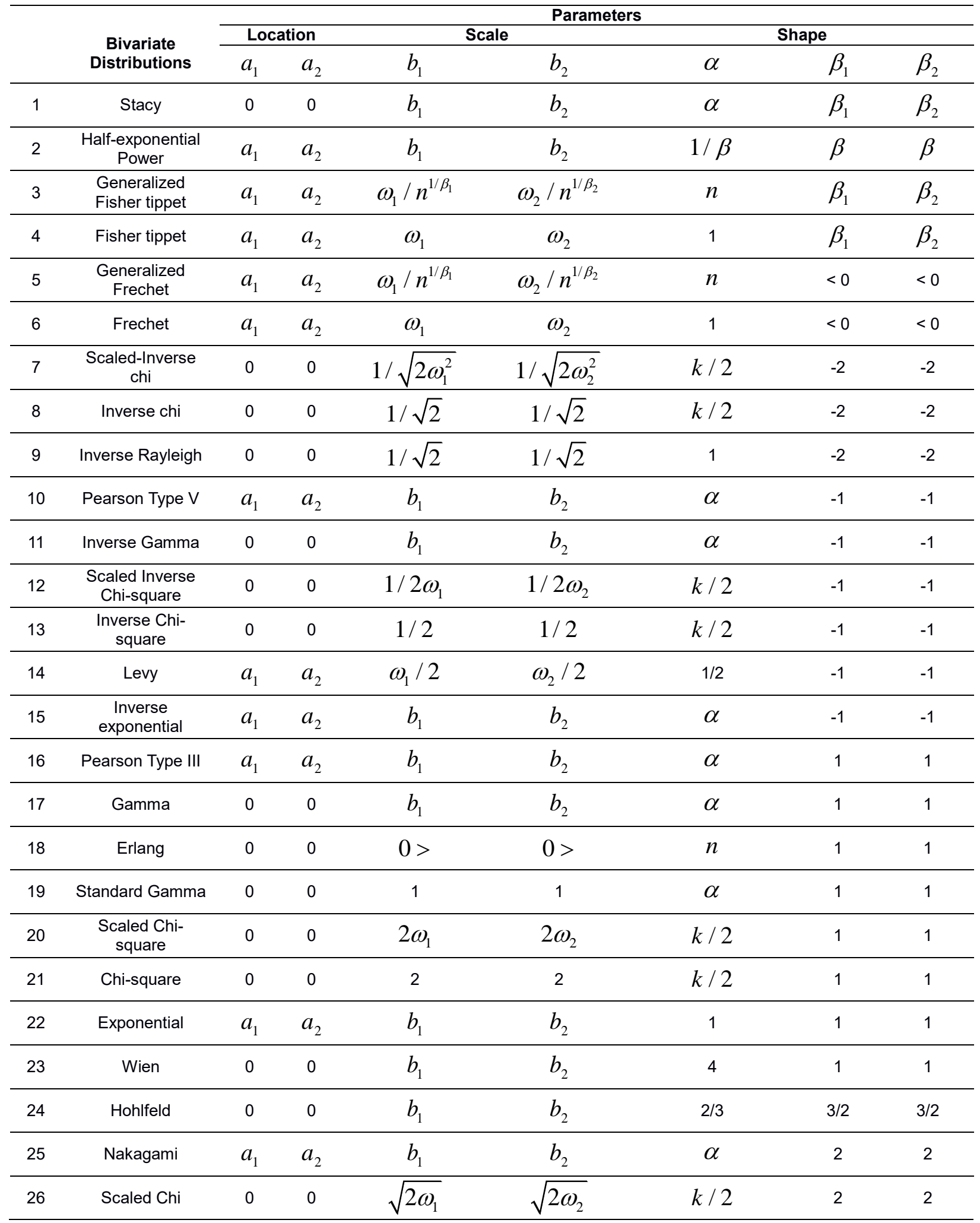




\begin{tabular}{|c|c|c|c|c|c|c|c|c|}
\hline & \multirow{3}{*}{$\begin{array}{l}\text { Bivariate } \\
\text { Distributions }\end{array}$} & \multicolumn{7}{|c|}{ Parameters } \\
\hline & & \multicolumn{2}{|c|}{ Location } & \multicolumn{2}{|c|}{ Scale } & \multicolumn{3}{|c|}{ Shape } \\
\hline & & $a_{1}$ & $a_{2}$ & $b_{1}$ & $b_{2}$ & $\alpha$ & $\beta_{1}$ & $\beta_{2}$ \\
\hline 27 & Chi & 0 & 0 & $\sqrt{2}$ & $\sqrt{2}$ & $k / 2$ & 2 & 2 \\
\hline 28 & Half normal & 0 & 0 & $\sqrt{2 \omega_{1}}$ & $\sqrt{2 \omega_{2}}$ & $1 / 2$ & 2 & 2 \\
\hline 29 & Rayleigh & 0 & 0 & $\sqrt{2 \omega_{1}}$ & $\sqrt{2 \omega_{2}}$ & 1 & 2 & 2 \\
\hline 30 & $\begin{array}{c}\text { Maxwell- } \\
\text { Boltzmann }\end{array}$ & 0 & 0 & $\sqrt{2 \omega_{1}}$ & $\sqrt{2 \omega_{2}}$ & $3 / 2$ & 2 & 2 \\
\hline 31 & Wilson- Hilferty & 0 & 0 & $b_{1}$ & $b_{2}$ & $\alpha$ & 3 & 3 \\
\hline 32 & $\begin{array}{c}\text { Generalized } \\
\text { Weibull }\end{array}$ & $a_{1}$ & $a_{2}$ & $\omega_{1} / n^{1 / \beta_{1}}$ & $\omega_{2} / n^{1 / \beta_{2}}$ & $n$ & $>0$ & $>0$ \\
\hline 33 & Weibul & $a_{1}$ & $a_{2}$ & $\omega_{1}$ & $\omega_{2}$ & 1 & $>0$ & $>0$ \\
\hline 34 & Pseudo-Weibull & $a_{1}$ & $a_{2}$ & $\omega_{1}$ & $\omega_{2}$ & $1+1 / \beta$ & $>0$ & $>0$ \\
\hline 35 & $\begin{array}{l}\text { Stretched } \\
\text { exponential }\end{array}$ & 0 & 0 & $b_{1}$ & $b_{2}$ & 1 & $>0$ & $>0$ \\
\hline 36 & Jeffreys & 0 & 0 & $b_{1}$ & $b_{2}$ & $\alpha$ & 0 & 0 \\
\hline 37 & log-gamma & $a_{1}$ & $a_{2}$ & $b_{1}$ & $b_{2}$ & $\alpha$ & $\lim _{\beta_{1} \rightarrow \infty}$ & $\lim _{\beta_{2} \rightarrow \infty}$ \\
\hline 38 & Power Law & $a_{1}$ & $a_{2}$ & $b_{1}$ & $b_{2}$ & $\delta /(1-\beta)$ & $\lim _{\beta_{1} \rightarrow \infty}$ & $\lim _{\beta_{2} \rightarrow \infty}$ \\
\hline 39 & log-normal & $a_{1}$ & $a_{2}$ & $b_{1}$ & $b_{2}$ & $1 /(\delta \beta)^{2}$ & $\lim _{\beta_{1} \rightarrow \infty}$ & $\lim _{\beta_{2} \rightarrow \infty}$ \\
\hline 40 & Normal & $a_{1}$ & $a_{2}$ & $b_{1}$ & $b_{2}$ & $\lim _{\alpha \rightarrow 0}$ & 1 & 1 \\
\hline
\end{tabular}


APPENDIX 2.

14. Bivariate Standard Levy distribution

$\left(\alpha=12, \beta_{1}=\beta_{2}=-1, \lambda=0.5\right) \quad\left(\alpha=1 / 2, \beta_{1}=\beta_{2}=-1, \lambda=-0.5\right)$
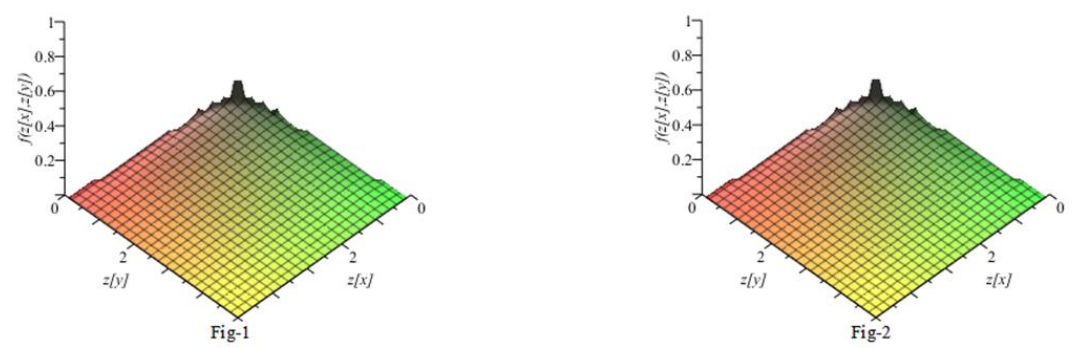

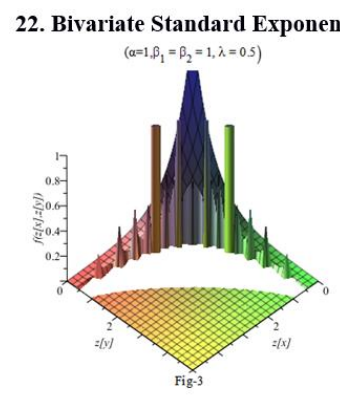

28. Bivariate Standard Half-normal distribution

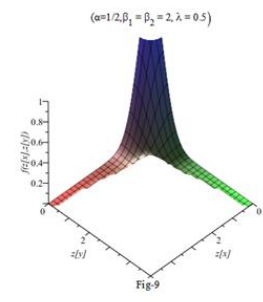

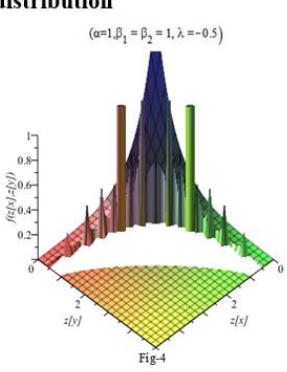

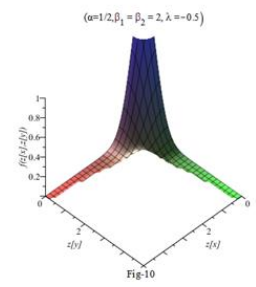

23. Bivariate Standard Wien distribution

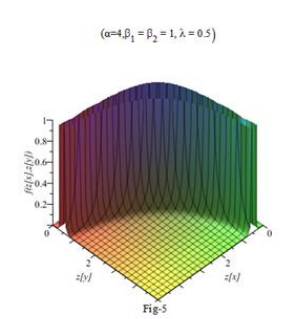

29. Bivariate Standard Rayleigh distribution

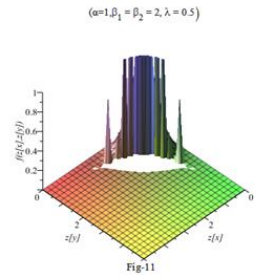

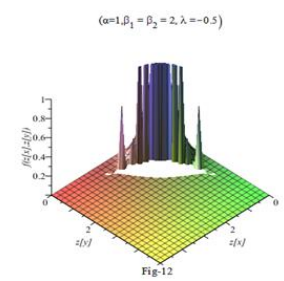

283
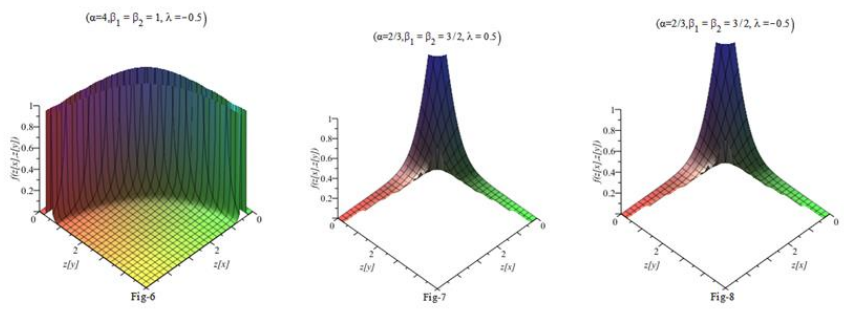

30. Bivariate Standard Maxwell-Boltzmann distribution
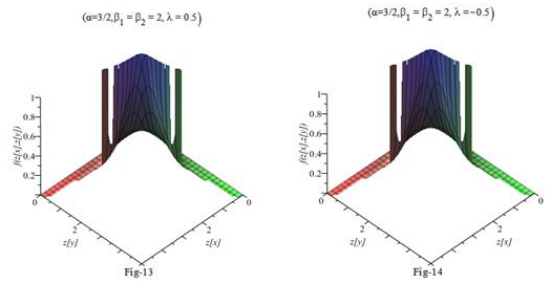